\title{
Antibacterial Compounds from Mushrooms: A Lead to Fight ESKAPEE Pathogenic Bacteria?
}

\author{
Authors \\ Violette Hamers, Clément Huguet, Mélanie Bourjot, Aurélie Urbain ${ }^{\mathbb{D}}$
}

\section{Affiliation}

Faculté de pharmacie, Université de Strasbourg, CNRS, IPHC UMR 7178, CAMBAP, Strasbourg, France

\section{Key words}

mushrooms, macromycetes, natural products, antibacterial, antibiotics, ESKAPEE

$\begin{array}{ll}\text { received } & \text { July } 20,2020 \\ \text { accepted after revision } & \text { September } 18,2020 \\ \text { published online } & \text { October } 15,2020\end{array}$

\section{Bibliography}

Planta Med 2021; 87: 351-367

DOI $10.1055 / a-1266-6980$

ISSN 0032-0943

(C) 2020. Thieme. All rights reserved.

Georg Thieme Verlag KG, Rüdigerstraße 14, 70469 Stuttgart, Germany

\section{Correspondence}

Dr Aurélie Urbain

Chimie Analytique des Molécules Bioactives et Pharmacognosie, IPHC UMR 7178, Université de Strasbourg 74 route du Rhin, CS 60024, 67401 Illkirch Cedex, France Phone: +33368854180 , Fax: +33368854325 aurbain@unistra.fr

Supplementary material is available under https://doi.org/10.1055/a-1266-6980

\section{ABSTRACT}

Infectious diseases are among the greatest threats to global health in the 21st century, and one critical concern is due to antibiotic resistance developed by an increasing number of bacterial strains. New resistance mechanisms are emerging with many infections becoming more and more difficult if not impossible to treat. This growing phenomenon not only is associated with increased mortality but also with longer hospital stays and higher medical costs. For these reasons, there is an urgent need to find new antibiotics targeting pathogenic microorganisms such as ESKAPEE bacteria. Most of currently approved antibiotics are derived from microorganisms, but higher fungi could constitute an alternative and remarkable reservoir of anti-infectious compounds. For instance, pleuromutilins constitute the first class of antibiotics derived from mushrooms. However, macromycetes still represent a largely unexplored source. Publications reporting the antibacterial potential of mushroom extracts are emerging, but few purified compounds have been evaluated for their bioactivity on pathogenic bacterial strains. Therefore, the aim of this review is to compile up-to-date data about natural products isolated from fruiting body fungi, which significantly inhibit the growth of ESKAPEE pathogenic bacteria. When available, data regarding modes of action and cytotoxicity, mandatory when considering a possible drug development, have been discussed in order to highlight the most promising compounds.

\section{Introduction}

Nowadays, 97\% of approved antibiotics have a microbial origin, from either soil fungi (e.g., penicillin) or bacteria (e.g., streptomycin) [1]. These antibacterial agents have revolutionized the treatment of infections such as tuberculosis, pneumonia, leprosy, and gonorrhea. Unfortunately, the overuse and misuse of antibiotics has gradually led to acquired drug resistance in bacterial strains, an ever-increasing phenomenon. Almost a century after Fleming's discovery of penicillin, there is a sore need to identify new antibacterial drugs capable of fighting infections caused by multidrug-resistant (MDR) bacteria.

Alternative sources could represent an interesting reservoir of such molecules. Over the past decade, search for antibacterial compounds has been extended to marine microorganisms or endophytic fungi, which also need to activate a whole antimicrobial chemical arsenal to impose themselves in their highly competitive environment [2,3]. In comparison to cultured bacteria and fungi, there has been considerably less attention devoted to investigating macroscopic fungi. Indeed, as they are also exposed to various biotic stresses, both at the underground (mycelium) and aerial (fruiting body) level, they synthesize a variety of antibacterial molecules. In Western countries, mushrooms are considered above all for their culinary and nutritional value, like truffles or boletus mushrooms that deliver a sought-after flavor, or white mushrooms, low in calories but providing a good source of dietary fibers, proteins, vitamins, and minerals. In Asia, where traditional Chinese medicine prevails, many mushrooms are not only popular 
ingredients in cuisine, but they are also reputed for their health benefits, like shiitake or Ganoderma lucidum, with numerous studies reporting their immunomodulatory properties and anticancer potential $[4,5]$. Yet, the interest in macromycetes as an alternative source of antibacterial agents seems to emerge somewhat $[6,7]$, and a new class of mushroom-derived antibiotics, pleuromutilins, has been released recently [8].

Although numerous recent publications report the antibacterial activity of mushroom extracts, studies dealing with purified and identified compounds are still scarce. Therefore, this review aims at establishing a state of the art on mushroom molecules that affect bacteria development. Some publications report antibacterial activity against nonpathogenic bacteria, such as the Gram-positive model Bacillus subtilis. We have considered that it would be much more valuable to pay attention to molecules inhibiting the growth of pathogenic bacteria only. For this reason, we have decided to focus this review on fungal compounds exhibiting an antibacterial activity toward bacteria that represent a major threat in this context of antibiotic resistance, namely ESKAPEE bacteria: Enterococcus faecium ( $\vee$ Table 1), Staphylococcus aureus ( $\vee$ Table 2), Klebsiella pneumoniae ( $\vee$ Table 3), Acinetobacter baumannii ( $\triangleright$ Table 4), Pseudomonas aeruginosa ( $\bullet$ Table 5$)$, Enterobacter species ( $\bullet$ Table 6 ), and Escherichia coli ( $\bullet$ Table 7) [9].

The applied search strategy consisted in the systematic literature reporting mushroom antibacterial compounds active against ESKAPEE bacteria, without any time limitation. Electronic databases including SciFinder, Web of Science, Science Direct, PubMed, and Google Scholar were screened using the following keywords: "antibacterial, antimicrobial, mushroom, fungi, compounds, metabolites, Enterococcus, Staphylococcus, Klebsiella, Acinetobacter, Pseudomonas, Enterobacter, Escherichia coli”. Careful attention was paid to select publications referring only to purified compounds isolated from macromycetes and evaluated against one or several of these 7 pathogenic strains.

\section{ESKAPEE Pathogenic Bacteria}

As mentioned above, the excessive or inappropriate use of antibiotics has progressively led to the emergence of multidrug resistance. After the golden age of antibiotics in the middle of the 20th century, bacteria began to evolve by developing a whole arsenal of resistance genes, leading, for example, to drug inactivation and biofilm formation [10]. As a result, MDR infections were responsible of more than 33000 deaths in Europe in 2015 [11], and in the United States, more than 2.8 million patients suffer from antibiotic-resistant infections each year [12]. Global data are alarming: drug-resistant infections are responsible for one death every $45 \mathrm{~s}$ in the world [13]. Besides this high increase in mortality and morbidity rates, antibiotic resistance also generates a high additional economic burden, reaching up to 20 billion dollars for the U.S. healthcare system $[12,14,15]$.

In 2017, the WHO published a list of antibiotic-resistant bacteria, divided into 3 categories according to their degree of pathogenicity and resistance; it consists of strains responsible for several difficult-to-treat infections in humans, including nosocomial infections [16]. The most critical bacteria associated with resistance and virulence are E. faecium, S. aureus, K. pneumoniae, A. bau- mannii, P. aeruginosa, and Enterobacter species, bacteria whose initials have given the acronym ESKAPE. This designation also highlights the ability of these pathogens to escape from many antibiotics, leading to severe diseases, such as bloodstream infections and pneumonia, associated with a high degree of mortality [17]. Although $E$. coli is not included in the ESKAPE group, this Gramnegative Enterobacteriaceae can produce enzymes involved in antibiotic resistance, such as carbapenemase and extended-spectrum beta-lactamases (ESBL). These resistance mechanisms make it a critical priority pathogen regarding the WHO list. On these arguments, we have decided to include in this review mushrooms metabolites that inhibit $E$. coli growth, leading to a review focusing on the extended ESKAPEE pathogenic bacteria.

Even if these microorganisms are often associated with MDR, we will see that many studies presented in this review have not necessarily been performed on resistant strains. For instance, all compounds reported to be active against $K$. pneumoniae, $P$. aeruginosa, and Enterobacter species have been evaluated on various strains not showing any special resistance to usual medication. On the contrary, all compounds inhibiting $E$. faecium growth have been tested on vancomycin-resistant strains. For S. aureus and E. coli, some molecules were active against ESBL-producing or methicillin-resistant strains; few metabolites were even active against a multiresistant $A$. baumannii strain. Some antibacterial assessments have also been carried out in the presence of phenylalanine-arginine $\beta$-naphthylamide (PA $\beta N$ ), an efflux inhibitor that permeabilizes the outer membrane of Gram-negative bacteria such as K. pneumoniae, A. baumannii, P. aeruginosa, and Enterobacter species, allowing bioactive compounds to reach their intracellular target. MIC and IC 50 presented in > Tables 1-7 are expressed in $\mu \mathrm{g} / \mathrm{mL}$, as recommended by standardized guidelines; however, for a better comparison of the efficacy of these secondary metabolites, these data are also available in $\mu \mathrm{M}$ (Table 1S, Supporting Information).

\section{Antibacterial Bioassays}

Various bioassays can be used to assess the antibacterial potential of extracts or single compounds [18]. The most widespread include microdilution techniques and agar diffusion methods, but bioautography on thin-layer chromatography, flow or image cytometry, or bioluminescence or biofilm assays can be also applied. Each of these techniques presents some advantages and drawbacks, but one of the main difficulties is to compare efficacy of compounds tested with different methods. Indeed, each method will lead to one or several specific measurement units. For example, antibacterial effect of a compound evaluated by disk diffusion method will be expressed in millimetres as the diameter of the growing inhibition zone; on the other hand, the antibacterial effect of the same compound tested with broth dilution method will be expressed as a concentration $(\mu \mathrm{g} / \mathrm{mL})$ that can refer to several different values:

i) the minimum concentration that inhibits the growth of the microorganism (MIC);

ii) the concentration required for inhibiting $50 \%$ of microbial growth $\left(\mathrm{IC}_{50}\right)$; 
- Table 1 Compounds isolated from mushrooms with antibacterial activity against Enterococcus faecium.

\begin{tabular}{|c|c|c|c|}
\hline $\begin{array}{l}\text { Chemical class } \\
\text { Compound }\end{array}$ & Inhibition & Source & Ref \\
\hline \multicolumn{4}{|l|}{ Diterpenoids } \\
\hline cyathinin A & $\mathrm{IC}_{50}=50 \mu \mathrm{g} / \mathrm{mL}^{\mathrm{a}}$ & \multirow{6}{*}{$\begin{array}{l}\text { Cyathus subglobisporus R. L. Zhao, Desjardin \& K. D. } \\
\text { Hyde (mycelium) }\end{array}$} & \multirow[t]{6}{*}{ [43] } \\
\hline cyathinin D & $\mathrm{IC}_{50}=50 \mu \mathrm{g} / \mathrm{mL}^{\mathrm{a}}$ & & \\
\hline striatal A & $\mathrm{IC}_{50}=25 \mu \mathrm{g} / \mathrm{mL}^{\mathrm{a}}$ & & \\
\hline striatal B & $\mathrm{IC}_{50}=6.25 \mu \mathrm{g} / \mathrm{mL}^{\mathrm{a}}$ & & \\
\hline striatal D & $\mathrm{IC}_{50}=50 \mu \mathrm{g} / \mathrm{mL}^{\mathrm{a}}$ & & \\
\hline striatin C & $\mathrm{IC}_{50}=25 \mu \mathrm{g} / \mathrm{mL}^{\mathrm{a}}$ & & \\
\hline \multicolumn{4}{|l|}{ Peptides } \\
\hline \multirow[t]{4}{*}{ copsin } & $\mathrm{MIC}=4 \mu \mathrm{g} / \mathrm{mL}$ & \multirow{4}{*}{$\begin{array}{l}\text { Coprinopsis cinerea (Schaeff.) Redhead, Vilgalys \& } \\
\text { Moncalvo (mycelium) }\end{array}$} & \multirow[t]{4}{*}{ [60] } \\
\hline & $\mathrm{MIC}=2 \mu \mathrm{g} / \mathrm{mL}^{\mathrm{b}}$ & & \\
\hline & $\mathrm{MBC}=8 \mu \mathrm{g} / \mathrm{mL}$ & & \\
\hline & $\mathrm{MBC}=2 \mu \mathrm{g} / \mathrm{mL}^{\mathrm{b}}$ & & \\
\hline \multirow[t]{2}{*}{ plectasin } & $\mathrm{MIC} \geq 16 \mu \mathrm{g} / \mathrm{mL}$ & \multirow[t]{2}{*}{ Pseudoplectania nigrella (Pers.) Fuckel (mycelium) } & \multirow[t]{2}{*}{ [57] } \\
\hline & $\mathrm{MIC} \geq 32 \mu \mathrm{g} / \mathrm{mL}^{\mathrm{b}}$ & & \\
\hline
\end{tabular}

iii) or the lowest concentration that kills $99.9 \%$ of the microorganisms, defined as the minimal bactericidal concentration (MBC), or more seldom the $\mathrm{IC}_{100}$ value.

The MIC value is generally preferred to $\mathrm{IC}_{50}$ to express antibacterial potential: although it is easy to observe the concentration that prevents bacterial development, it is harder to calculate thoroughly the concentration inhibiting the growth of half of the bacteria. Microdilution methods generally consist of series of 2-fold dilutions, and the inhibitory effect is usually observed over a very narrow concentration range. Consequently, a graph plotting bacterial growth versus concentration of the active compound may be too steep to calculate the $\mathrm{IC}_{50}$ value accurately.

Concerning the agar method, this one can be carried out by depositing the compound to be evaluated either in a well dug in the agar sterilely, or on a paper disc brought into contact with the agar. In any cases, only few publications mention the diameter of the disc or well, yet this is fundamental for determining whether an inhibition diameter is substantial or not. If a zone of inhibition measures $14 \mathrm{~mm}$, it does not have the same meaning with a disk of 6 , 9 , or $12 \mathrm{~mm}$ of diameter. In addition, it is important to be precise as to whether the whole diameter is measured, including the central disk, or if the measurement is done from the edge of the paper disk. For all these reasons, it can be challenging to discuss the antibacterial potential of compounds when experimental procedure is not clearly mentioned, as well as results for a positive control.

Antibacterial activity of mushroom compounds presented in this review ( $\vee$ Tables $1-\mathbf{7}$ ) are expressed either as growth inhibition zone diameter (ZI), half maximal inhibitory concentration $\left(\mathrm{IC}_{50}\right)$, minimum inhibitory concentration (MIC), or minimum bactericidal concentration (MBC). Regarding diffusion assays, values were obtained for a quantity of $50 \mu \mathrm{g}$ of compound, unless other- wise stated. Most of the inhibitory values presented herein have been determined following validated methods, such as Clinical \& Laboratory Standards Institute (CLSI) guidelines or methods described by the European Committee on Antimicrobial Susceptibility Testing (EUCAST). As the aim of this review is to highlight the potential of fungal metabolites to inhibit the growth of ESKAPEE bacteria, we have decided to set a threshold in order to select the more interesting compounds in terms of activity: only metabolites exhibiting an MIC below or equal to $250 \mu \mathrm{g} / \mathrm{mL}$ toward sensitive strains have been retained (for $I C_{50}$, no value exceeds $50 \mu \mathrm{g} /$ $\mathrm{mL}$ ). When data were also available for resistant strains, they have been included even if values were then slightly higher. We have selected this maximal value of $250 \mu \mathrm{g} / \mathrm{mL}$ based on the MIC distribution data collected by EUCAST, reporting modal MIC around $100 \mathrm{\mu g} / \mathrm{mL}$ for several bacteria, such as $K$. pneumoniae, E. coli, and Enterobacter spp. [19]. Concerning diffusion methods, the minimum threshold diameter has been set to $12 \mathrm{~mm}$ for $50 \mu \mathrm{g}$ of compound. However, these values should not be taken at face value. For example, according to the EUCAST guidelines, Enterobacter species are considered to be sensitive to ampicillin if the inhibition diameter is higher than $14 \mathrm{~mm}$ but sensitive to imipenem only if the diameter is larger than $50 \mathrm{~mm}$ [19].

We have also reckoned that activity observed for high amounts of compounds only could be associated with cytotoxicity toward eukaryotic cells; yet efficacy and safety are mandatory for considering an antibacterial compound as a potential antibiotic-candidate. While most of the publications do not mention anything regarding potential cytotoxicity, some authors have investigated both antibacterial and cytotoxic effects. These data are discussed below, as well as the mechanisms of action when appropriate. 
- Table 2 Compounds isolated from mushrooms with antibacterial activity against Staphylococcus aureus.

\begin{tabular}{|c|c|c|c|}
\hline $\begin{array}{l}\text { Chemical class } \\
\text { Compound }\end{array}$ & Inhibition & Source & Ref \\
\hline \multicolumn{4}{|l|}{ Anthraquinones } \\
\hline (1S,3R)-austrocortilutein & $\mathrm{IC}_{50}=12 \mu \mathrm{g} / \mathrm{mL}$ & \multirow{6}{*}{$\begin{array}{l}\text { Cortinarius basirubescens Cleland \& J. R. Harris } \\
\text { (fruiting body) }\end{array}$} & \multirow[t]{6}{*}[20]{} \\
\hline (1S,3S)-austrocortilutein & $\mathrm{IC}_{50}=8 \mu \mathrm{g} / \mathrm{mL}$ & & \\
\hline$(1 \mathrm{~S}, 3 \mathrm{~S})$-austrocortirubin & $\mathrm{IC}_{50}=3 \mu \mathrm{g} / \mathrm{mL}$ & & \\
\hline Torosachrysone & $\mathrm{IC}_{50}=10 \mu \mathrm{g} / \mathrm{mL}$ & & \\
\hline Emodin & $\mathrm{IC}_{50}=0.7 \mu \mathrm{g} / \mathrm{mL}$ & & \\
\hline parietin (physcion) & $\begin{array}{l}\mathrm{IC}_{50}=23 \mu \mathrm{g} / \mathrm{mL} \\
\mathrm{MIC}=0.5 \mu \mathrm{g} / \mathrm{mL}\end{array}$ & & \\
\hline \multicolumn{4}{|l|}{ Pyranoquinones } \\
\hline multiformin A & $\mathrm{ZI}=18 \mathrm{~mm}$ & \multirow[t]{4}{*}{ Hypoxylon multiforme (Fr.) Fr. (stromata) } & \multirow[t]{4}{*}[25]{} \\
\hline multiformin B & $\mathrm{ZI}=20 \mathrm{~mm}$ & & \\
\hline multiformin $C$ & $\mathrm{ZI}=18 \mathrm{~mm}$ & & \\
\hline multiformin D & $\mathrm{ZI}=15 \mathrm{~mm}$ & & \\
\hline sassafrin A & $\mathrm{ZI}=19 \mathrm{~mm}$ & \multirow{4}{*}{$\begin{array}{l}\text { Creosphaeria sassafras (Schwein.) Y. M. Ju, F. San } \\
\text { Martín \&J. D. Rogers (stromata) }\end{array}$} & \multirow[t]{4}{*}[24]{} \\
\hline sassafrin B & $\mathrm{ZI}=18 \mathrm{~mm}$ & & \\
\hline sassafrin C & $\mathrm{ZI}=22 \mathrm{~mm}$ & & \\
\hline sassafrin D & $\mathrm{ZI}=17 \mathrm{~mm}$ & & \\
\hline \multicolumn{4}{|l|}{ Terphenyl quinone } \\
\hline spiromentin C & $\mathrm{MIC}=250 \mu \mathrm{g} / \mathrm{mL}^{\mathrm{a}}$ & $\begin{array}{l}\text { Tapinella atrotomentosa (Batsch) Šutara (fruiting } \\
\text { body) }\end{array}$ & {$[27]$} \\
\hline \multicolumn{4}{|l|}{ Lactone derivatives } \\
\hline Osmundalactone & $\mathrm{MIC}=250 \mu \mathrm{g} / \mathrm{mL}^{\mathrm{a}}$ & \multirow{2}{*}{$\begin{array}{l}\text { Tapinella atrotomentosa (Batsch) Šutara (fruiting } \\
\text { body) }\end{array}$} & \multirow[t]{2}{*}[27]{} \\
\hline 5-hydroxy-hex-2-en-4-olide & $\mathrm{MIC}=250 \mu \mathrm{g} / \mathrm{mL}^{\mathrm{a}}$ & & \\
\hline coloratin A & $\mathrm{Zl}=15 \mathrm{~mm}$ & $\begin{array}{l}\text { Xylaria intracolorata (J. D. Rogers, Callan \& Sa- } \\
\text { muels) J. D. Rogers \& Y. M. Ju (stromata) }\end{array}$ & {$[76]$} \\
\hline skeletocutin A & $\begin{array}{l}\mathrm{MIC}=37.5 \mu \mathrm{g} / \mathrm{mL} \\
\mathrm{MIC}=150 \mu \mathrm{g} / \mathrm{mL}^{\mathrm{b}}\end{array}$ & \multirow[t]{9}{*}{ Skeletocutis sp. (fruiting body) } & \multirow[t]{9}{*}{ [32] } \\
\hline skeletocutin B & $\begin{array}{l}\mathrm{MIC}=150 \mu \mathrm{g} / \mathrm{mL} \\
\mathrm{MIC}=300 \mu \mathrm{g} / \mathrm{mL}^{\mathrm{b}}\end{array}$ & & \\
\hline skeletocutin C & $\begin{array}{l}\mathrm{MIC}=150 \mu \mathrm{g} / \mathrm{mL} \\
\mathrm{MIC}=300 \mu \mathrm{g} / \mathrm{mL}^{\mathrm{b}}\end{array}$ & & \\
\hline skeletocutin D & $\begin{array}{l}\mathrm{MIC}=150 \mu \mathrm{g} / \mathrm{mL} \\
\mathrm{MIC}=300 \mu \mathrm{g} / \mathrm{mL}^{\mathrm{b}}\end{array}$ & & \\
\hline skeletocutin E & $\begin{array}{l}\mathrm{MIC}=37.5 \mu \mathrm{g} / \mathrm{mL} \\
\mathrm{MIC}=150 \mu \mathrm{g} / \mathrm{mL}^{\mathrm{b}}\end{array}$ & & \\
\hline skeletocutin J & $\begin{array}{l}\mathrm{MIC}=150 \mu \mathrm{g} / \mathrm{mL} \\
\mathrm{MIC}=150 \mu \mathrm{g} / \mathrm{mL}^{\mathrm{b}}\end{array}$ & & \\
\hline skeletocutin K & $\begin{array}{l}\mathrm{MIC}=37.5 \mu \mathrm{g} / \mathrm{mL} \\
\mathrm{MIC}=75 \mu \mathrm{g} / \mathrm{mL}^{\mathrm{b}}\end{array}$ & & \\
\hline skeletocutin L & $\begin{array}{l}\mathrm{MIC}=18.75 \mu \mathrm{g} / \mathrm{mL} \\
\mathrm{MIC}=75 \mu \mathrm{g} / \mathrm{mL}^{\mathrm{b}}\end{array}$ & & \\
\hline tyromycin A & $\begin{array}{l}\mathrm{MIC}=150 \mu \mathrm{g} / \mathrm{mL} \\
\mathrm{MIC}=150 \mu \mathrm{g} / \mathrm{mL}^{\mathrm{b}}\end{array}$ & & \\
\hline \multicolumn{4}{|l|}{ Furan derivative } \\
\hline 5-hydroxy-methylfurfural & $\begin{array}{l}\mathrm{ZI}=28 \mathrm{~mm} \\
\mathrm{MIC}=250 \mu \mathrm{g} / \mathrm{mL}\end{array}$ & $\begin{array}{l}\text { Morchella esculenta (L.) Pers./Verpa bohemica } \\
\text { (Krombh.) J. Schröt. (fruiting body) }\end{array}$ & $\begin{array}{l}{[37]} \\
\text { cont }\end{array}$ \\
\hline
\end{tabular}


- Table 2 Continued

\begin{tabular}{|c|c|c|c|}
\hline $\begin{array}{l}\text { Chemical class } \\
\text { Compound }\end{array}$ & Inhibition & Source & Ref \\
\hline \multicolumn{4}{|l|}{ Phenolic acids } \\
\hline cinnamic acid & $\mathrm{MIC}=1.5 \mu \mathrm{g} / \mathrm{mL}$ & \multirow{2}{*}{$\begin{array}{l}\text { Ganoderma lucidum (Curtis) P. Karst. } \\
\text { (fruiting body) }\end{array}$} & \multirow[t]{2}{*}{ [35] } \\
\hline p-hydroxybenzoic acid & $\mathrm{MIC}=3 \mu \mathrm{g} / \mathrm{mL}$ & & \\
\hline \multicolumn{4}{|l|}{ Sesquiterpenoids } \\
\hline 7-acetyl-4-methyl-azulene-1-carbaldehyde & $\mathrm{ZI}=15 \mathrm{~mm}$ & Lactarius deliciosus (L.) Gray (fruiting body) & [77] \\
\hline enokipodin A & $\mathrm{ZI}=25.2 \mathrm{~mm}$ & \multirow[t]{3}{*}{ Flammulina velutipes (Curtis) Singer (mycelium) } & \multirow[t]{3}{*}[40]{} \\
\hline enokipodin B & $\mathrm{ZI}=14 \mathrm{~mm}$ & & \\
\hline enokipodin C & $\mathrm{ZI}=21 \mathrm{~mm}$ & & \\
\hline phellodonic acid & $\mathrm{MIC}=10 \mu \mathrm{g} / \mathrm{mL}$ & $\begin{array}{l}\text { Phellodon melaleucus (Sw. ex Fr.) P. Karst. } \\
\text { (mycelium) }\end{array}$ & [39] \\
\hline stereumamide $\mathrm{A}$ & $\mathrm{MIC}=12.5 \mu \mathrm{g} / \mathrm{mL}$ & \multirow[t]{3}{*}{ Stereum hirsutum (Willd.) Pers. (mycelium) } & \multirow[t]{3}{*}{ [38] } \\
\hline stereumamide D & $\mathrm{MIC}=12.5 \mu \mathrm{g} / \mathrm{mL}$ & & \\
\hline sterostrein Q & $\mathrm{MIC}=25 \mu \mathrm{g} / \mathrm{mL}$ & & \\
\hline \multicolumn{4}{|l|}{ Diterpenoids } \\
\hline psathyrelloic acid & $\mathrm{MIC}=16 \mu \mathrm{g} / \mathrm{mL}$ & Psathyrella candolleana (Fr.) Maire (mycelium) & [46] \\
\hline sarcodonin L & $\mathrm{ZI}=18 \mathrm{~mm}$ with $35 \mu \mathrm{g}$ & \multirow[t]{2}{*}{ Sarcodon scabrosus (Fr.) P. Karst. (fruiting body) } & \multirow[t]{2}{*}{ [45] } \\
\hline sarcodonin M & $\mathrm{ZI}=22 \mathrm{~mm}$ with $35 \mu \mathrm{g}$ & & \\
\hline psathyrin A & $\mathrm{MIC}=14.3 \mu \mathrm{g} / \mathrm{mL}$ & \multirow[t]{2}{*}{ Psathyrella candolleana (Fr.) Maire (mycelium) } & \multirow[t]{2}{*}{ [47] } \\
\hline psathyrin B & $\mathrm{MIC}=22.7 \mu \mathrm{g} / \mathrm{mL}$ & & \\
\hline \multicolumn{4}{|l|}{ Triterpenoids } \\
\hline ganoderic acid & $\mathrm{MIC}=250 \mu \mathrm{g} / \mathrm{mL}$ & Ganoderma lucidum (Curtis) P. Karst. (mycelium) & [36] \\
\hline \multicolumn{4}{|l|}{ Meroterpenoids } \\
\hline ganomycin A & $\mathrm{MIC}=25 \mu \mathrm{g} / \mathrm{mL}$ & \multirow[t]{2}{*}{ Ganoderma pfeifferi Bres. (fruiting body) } & \multirow[t]{2}{*}{ [42] } \\
\hline ganomycin B & $\mathrm{MIC}=25 \mu \mathrm{g} / \mathrm{mL}$ & & \\
\hline \multicolumn{4}{|l|}{ Steroid } \\
\hline ergosterol & $\begin{array}{l}\mathrm{ZI}=25 \mathrm{~mm} \\
\mathrm{MIC}=250 \mu \mathrm{g} / \mathrm{mL}\end{array}$ & $\begin{array}{l}\text { Morchella esculenta (L.) Pers./Verpa bohemica } \\
\text { (Krombh.) J. Schröt. (fruiting body) }\end{array}$ & [37] \\
\hline \multicolumn{4}{|l|}{ Alkaloids (piperazine derivatives) } \\
\hline emestrin & $\begin{array}{l}\mathrm{IC}_{50}=4.55 \mu \mathrm{g} / \mathrm{mL} \\
\mathrm{IC} \mathrm{C}_{50}=2.21 \mu \mathrm{g} / \mathrm{mL}^{\mathrm{b}} \\
\mathrm{MIC}=20 \mu \mathrm{g} / \mathrm{mL} \\
\mathrm{MIC}=10 \mu \mathrm{g} / \mathrm{mL}^{\mathrm{b}}\end{array}$ & $\begin{array}{l}\text { Desarmillaria tabescens (Scop.) R. A. Koch \& Aime } \\
\text { (mycelium) }\end{array}$ & [55] \\
\hline epicorazine A & $\mathrm{ZI}=35 \mathrm{~mm}$ with $100 \mu \mathrm{g}$ & \multirow[t]{3}{*}{ Podaxis pistillaris (L.) Fr. (mycelium) } & \multirow[t]{3}{*}{ [54] } \\
\hline epicorazine B & $\mathrm{ZI}=30 \mathrm{~mm}$ with $100 \mu \mathrm{g}$ & & \\
\hline epicorazine C & $\mathrm{ZI}=25 \mathrm{~mm}$ with $100 \mu \mathrm{g}$ & & \\
\hline \multicolumn{4}{|l|}{ Sulfur derivative } \\
\hline bis((methylsulfonyl) methyl) disulfide & $\mathrm{MIC}=12.5 \mu \mathrm{g} / \mathrm{mL}$ & Lentinus edodes (Berk.) Singer (fruiting body) & [73] \\
\hline \multicolumn{4}{|l|}{ Fatty acid } \\
\hline pentadecanoic acid & $\begin{array}{l}\mathrm{ZI}=17 \mathrm{~mm} \\
\mathrm{MIC}=250 \mu \mathrm{g} / \mathrm{mL}\end{array}$ & $\begin{array}{l}\text { Morchella esculenta (L.) Pers./Verpa bohemica } \\
\text { (Krombh.) J. Schröt. (fruiting body) }\end{array}$ & [37] \\
\hline \multicolumn{4}{|l|}{ Polyacetylene } \\
\hline drosophilin D & $\mathrm{MIC}=4 \mu \mathrm{g} / \mathrm{mL}$ & Drosophila subatrata (Batsch) Quél. (mycelium) & [50] \\
\hline \multicolumn{4}{|l|}{ Polysaccharide } \\
\hline polysaccharide PL2 & $\mathrm{MIC}=25 \mu \mathrm{g} / \mathrm{mL}$ & $\begin{array}{l}\text { Lentinus edodes (Berk.) Singer (spent mushroom } \\
\text { substrate) }\end{array}$ & [66] \\
\hline
\end{tabular}


- Table 2 Continued

\begin{tabular}{|c|c|c|c|}
\hline $\begin{array}{l}\text { Chemical class } \\
\text { Compound }\end{array}$ & Inhibition & Source & Ref \\
\hline \multicolumn{4}{|l|}{ Peptides } \\
\hline boletusin & $13 \leq \mathrm{ZI} \leq 15 \mathrm{~mm}$ & \multirow[t]{3}{*}{ Boletus sp. (fruiting body) } & \multirow[t]{3}{*}{61} \\
\hline chrysospermin B & $14 \leq \mathrm{ZI} \leq 16 \mathrm{~mm}$ & & \\
\hline chrysospermin D & $13 \leq \mathrm{ZI} \leq 15 \mathrm{~mm}$ & & \\
\hline plectasin & $\begin{array}{l}4 \leq \mathrm{MIC} \leq 32 \mu \mathrm{g} / \mathrm{mL} \\
16 \leq \mathrm{MIC} \leq 32 \mu \mathrm{g} / \mathrm{mL}^{\mathrm{b}}\end{array}$ & Pseudoplectania nigrella (Pers.) Fuckel (mycelium) & {$[57$} \\
\hline \multicolumn{4}{|l|}{ Proteins } \\
\hline Cordyceps sinensis antibacterial protein & $50<\mathrm{MlC}<75 \mu \mathrm{g} / \mathrm{mL}$ & Cordyceps sinensis (Berk.) Sacc. (mycelium) & {$[62$} \\
\hline F2 protein & $\mathrm{IC}_{50}<100 \mu \mathrm{g} / \mathrm{mL}^{\mathrm{b}}$ & $\begin{array}{l}\text { Agaricus bisporus (J. E. Lange) Imbach } \\
\text { (fruiting body) }\end{array}$ & {$[63]$} \\
\hline
\end{tabular}

- Table 3 Compounds isolated from mushrooms with antibacterial activity against Klebsiella pseumoniae.

\begin{tabular}{|c|c|c|c|}
\hline $\begin{array}{l}\text { Chemical class } \\
\text { Compound }\end{array}$ & Inhibition & Source & Ref \\
\hline \multicolumn{4}{|l|}{ Pyranoquinones } \\
\hline multiformin A & $\mathrm{ZI}=18 \mathrm{~mm}$ & \multirow[t]{4}{*}{ Hypoxylon multiforme (Fr.) Fr. (stromata) } & \multirow[t]{4}{*}{ [25] } \\
\hline multiformin B & $\mathrm{Zl}=20 \mathrm{~mm}$ & & \\
\hline multiformin C & $\mathrm{ZI}=18 \mathrm{~mm}$ & & \\
\hline multiformin D & $\mathrm{ZI}=16 \mathrm{~mm}$ & & \\
\hline sassafrin A & $\mathrm{Zl}=20 \mathrm{~mm}$ & \multirow{4}{*}{$\begin{array}{l}\text { Creosphaeria sassafras (Schwein.) Y. M. Ju, F. San Martín \& J. D. } \\
\text { Rogers (stromata) }\end{array}$} & \multirow[t]{4}{*}{ [24] } \\
\hline sassafrin B & $\mathrm{ZI}=20 \mathrm{~mm}$ & & \\
\hline sassafrin C & $\mathrm{ZI}=22 \mathrm{~mm}$ & & \\
\hline sassafrin D & $\mathrm{ZI}=17 \mathrm{~mm}$ & & \\
\hline \multicolumn{4}{|c|}{ Indole-quinone polymer } \\
\hline Melanin & $\mathrm{ZI}=25 \mathrm{~mm}$ & Schizophyllum commune Fr. (mycelium) & [78] \\
\hline \multicolumn{4}{|l|}{ Aromatic lactone } \\
\hline coloratin A & $\mathrm{ZI}=22 \mathrm{~mm}$ & $\begin{array}{l}\text { Xylaria intracolorata (J. D. Rogers, Callan \& Samuels) J. D. } \\
\text { Rogers \& Y. M. Ju (stromata) }\end{array}$ & [76] \\
\hline \multicolumn{4}{|l|}{ Diterpenoids } \\
\hline pleuromutilin & $\mathrm{MIC}=1 \mu \mathrm{g} / \mathrm{mL}$ & Pleurotus passeckerianus Pilát (mycelium) & [48] \\
\hline striatal A & $1 C_{50}=50 \mu \mathrm{g} / \mathrm{mL}^{\mathrm{a}}$ & \multirow{3}{*}{$\begin{array}{l}\text { Cyathus subglobisporus R. L. Zhao, Desjardin \& K. D. Hyde } \\
\text { (mycelium) }\end{array}$} & \multirow[t]{3}{*}[43]{} \\
\hline striatal B & $\mathrm{IC}_{50}=25 \mu \mathrm{g} / \mathrm{mL}^{\mathrm{a}}$ & & \\
\hline striatin C & $\mathrm{IC}_{50}=50 \mu \mathrm{g} / \mathrm{mL}^{\mathrm{a}}$ & & \\
\hline \multicolumn{4}{|c|}{ Chlorinated derivative } \\
\hline drosophilin A & $\mathrm{MIC}=64 \mu \mathrm{g} / \mathrm{mL}$ & Drosophila subatrata (Batsch) Quél. (mycelium) & [50] \\
\hline \multicolumn{4}{|l|}{ Polysaccharides } \\
\hline exopolysaccharides & $\mathrm{MIC}=9.2 \mu \mathrm{g} / \mathrm{mL}$ & Lentinus edodes (Berk.) Singer (mycelium) & [65] \\
\hline
\end{tabular}


- Table 4 Compounds isolated from mushrooms with antibacterial activity against Acinetobacter baumannii.

\begin{tabular}{|c|c|c|c|}
\hline $\begin{array}{l}\text { Chemical class } \\
\text { Compound }\end{array}$ & Inhibition & Source & Ref \\
\hline \multicolumn{4}{|l|}{ Lactone derivatives } \\
\hline 5-hydroxy-hex-2-en-4-olide & $\mathrm{MIC}=6 \mu \mathrm{g} / \mathrm{mL}^{\mathrm{a}}$ & \multirow[t]{2}{*}{ Tapinella atrotomentosa (Batsch) Šutara (fruiting body) } & \multirow[t]{2}{*}{ [27] } \\
\hline Osmundalactone & $\mathrm{MIC}=10 \mu \mathrm{g} / \mathrm{mL}^{\mathrm{a}}$ & & \\
\hline \multicolumn{4}{|l|}{ Terphenyl quinone } \\
\hline spiromentin C & $\mathrm{MIC}=20 \mu \mathrm{g} / \mathrm{mL}^{\mathrm{a}}$ & Tapinella atrotomentosa (Batsch) Šutara (fruiting body) & [27] \\
\hline \multicolumn{4}{|l|}{ Diterpenoids } \\
\hline cyathinin D & $\mathrm{IC}_{50}=6.25 \mu \mathrm{g} / \mathrm{mL}^{\mathrm{b}}$ & \multirow{5}{*}{$\begin{array}{l}\text { Cyathus subglobisporus R. L. Zhao, Desjardin \& K. D. } \\
\text { Hyde (mycelium) }\end{array}$} & \multirow[t]{5}{*}{ [43] } \\
\hline striatal A & $\mathrm{IC}_{50}=12.5 \mu \mathrm{g} / \mathrm{mL}^{\mathrm{b}}$ & & \\
\hline striatal B & $\mathrm{IC}_{50}=6.25 \mu \mathrm{g} / \mathrm{mL}^{\mathrm{b}}$ & & \\
\hline striatin C & $\mathrm{IC}_{50}=3.13 \mu \mathrm{g} / \mathrm{mL}^{\mathrm{b}}$ & & \\
\hline striatoid C & $\mathrm{IC}_{50}=12.5 \mu \mathrm{g} / \mathrm{mL}^{\mathrm{b}}$ & & \\
\hline \multicolumn{4}{|l|}{ Alkaloid } \\
\hline 2-aminoquinoline & $\mathrm{MIC}=128 \mu \mathrm{g} / \mathrm{mL}$ & Leucopaxillus albissimus (Peck) Singer (fruiting body) & [79] \\
\hline
\end{tabular}

\section{Antibacterial Mushrooms Metabolites}

In recent decades, the antimicrobial potential of mushrooms has been highlighted, and several antibacterial compounds have been reported, mostly from higher basidiomycetes and some ascomycetes. Active compounds are mainly secondary metabolites (terpenoids, quinone or lactone derivatives, alkaloids, etc.), but high molecular weight molecules like polysaccharides or proteins seem to contribute as well to anti-infectious properties of mushrooms. Some chlorinated and sulfurated derivatives, often encountered in macromycetes, are also parts of the bioactive compounds. The collected data are presented in $\boldsymbol{\sim}$ Tables 1-7 for each targeted ESKAPEE bacteria, and some structures of bioactive compounds are presented in $\boldsymbol{\nabla}$ Fig. $\mathbf{1}$.

\section{Quinone Derivatives}

A large part of antibacterial compounds identified in macromycetes are quinones and their derivatives, known to exhibit antimicrobial activity. Major antibacterial quinoids from macrofungi are anthraquinone and pyranoquinone derivatives, but some $p$ terphenyl quinones and indole-quinone polymers (melanins) have also been reported to have antibacterial activities.

Beattie et al. [20] described the purification of 8 antibacterial anthraquinoid pigments from Cortinarius basirubescens (Cortinariaceae); emodin shows the best antibacterial activity against both $S$. aureus and $P$. aeruginosa, with $I C_{50}$ values of 0.7 and $1.5 \mu \mathrm{g} / \mathrm{mL}$, respectively. On the other hand, torosachrysone, austrocortirubin, and austrocortiluteins possess more specific antibacterial activity against $S$. aureus, contrary to 6-methylxanthopurpurin-3-0-methyl ether, which displays more remarkable activity against $P$. aeruginosa than against $S$. aureus. Parietin (1) found in Cortinarius species is also a potent growth inhibitor of S. aureus and P. aeruginosa (MIC of $0.5 \mu \mathrm{g} / \mathrm{mL}$ ), notably acting through photosensitized generation of ${ }^{1} \mathrm{O}_{2}$ and superoxide radical anion $\mathrm{O}_{2}{ }^{\bullet-}[20,21]$. In another study, the antibiofilm activity of parietin has been demonstrated against $S$. aureus and $E$. faecalis [22], which is particularly interesting as biofilms are implicated in $80 \%$ of human microbial infections. Generally speaking, anthraquinone derivatives-also widespread in plants-are well-known for their antimicrobial properties, and several mechanisms have been already identified [23], but they are also known for their toxic and laxative effects, which might cause undesirable side effects if drug development would be considered. Therefore, a full set of cytotoxic experiments should be consistently performed to confirm both antibiotic potential and safety of anthraquinone derivatives.

The fungal pyranoquinones multiformins and sassafrins (azaphilones) isolated from the Ascomycetes Hypoxylon multiforme and Creosphaeria sassafras, respectively, also possess antibacterial activities [24,25]. In a disk diffusion assay, they display moderate antibacterial activity against $S$. aureus, E. coli, P. aeruginosa, and K. pneumoniae when tested at $50 \mu \mathrm{g}$. Sassafrin C (2) followed by multiformin $B$ show the best inhibition activity against all tested bacteria, even though the inhibition diameters of all azaphilones are below the values of antibiotic standards. Interestingly, multiformin $D$ displays bacteriostatic effect against $S$. aureus, P. aeruginosa, and $E$. coli and has a weak bactericidal effect against $K$. pneumoniae. However, cytotoxic activities of some sassafrin and multiformin azaphilones have been reported at concentrations up to $30 \mu \mathrm{M}[26]$.

Spiromentin C (3), a $p$-terphenyl quinone purified from the velvet roll-rim Tapinella atrotomentosa (Tapinellaceae, Basidiomycetes), exerts an interesting inhibition against ESBL-producing E. coli, as well as against multiresistant $A$. baumannii, with MIC values of 10 and $20 \mu \mathrm{g} / \mathrm{mL}$, respectively [27]. Even though A. baumannii is defined as sensitive to conventional antibiotics for MIC below $8 \mu \mathrm{g} / \mathrm{mL}$ [18], this result is nevertheless encouraging, as 
- Table 5 Compounds isolated from mushrooms with antibacterial activity against Pseudomonas aeruginosa.

\begin{tabular}{|c|c|c|c|}
\hline $\begin{array}{l}\text { Chemical class } \\
\text { Compound }\end{array}$ & Inhibition & Source & Ref \\
\hline \multicolumn{4}{|l|}{ Anthraquinones } \\
\hline 6-methylxantho-purpurin-3-0-methyl ether & $\mathrm{IC} 50=31 \mu \mathrm{g} / \mathrm{mL}$ & \multirow{4}{*}{$\begin{array}{l}\text { Cortinarius basirubescens Cleland \& J.R. Harris } \\
\text { (fruiting body) }\end{array}$} & \multirow[t]{3}{*}[20]{} \\
\hline Emodin & $\mathrm{IC}_{50}=1.5 \mu \mathrm{g} / \mathrm{mL}$ & & \\
\hline parietin (physcion) & $\mathrm{I} \mathrm{C}_{50}=2 \mu \mathrm{g} / \mathrm{mL}$ & & \\
\hline parietin (physcion) & $\mathrm{MIC}=0.5 \mu \mathrm{g} / \mathrm{mL}$ & & {$[21]$} \\
\hline \multicolumn{4}{|l|}{ Pyranoquinones } \\
\hline sassafrin A & $\mathrm{ZI}=18 \mathrm{~mm}$ & \multirow{4}{*}{$\begin{array}{l}\text { Creosphaeria sassafras (Schwein.) Y. M. Ju, F. San } \\
\text { Martín \&J. D. Rogers (stromata) }\end{array}$} & \multirow[t]{4}{*}[24]{} \\
\hline sassafrin B & $\mathrm{ZI}=19 \mathrm{~mm}$ & & \\
\hline sassafrin C & $\mathrm{ZI}=22 \mathrm{~mm}$ & & \\
\hline sassafrin D & $\mathrm{ZI}=19 \mathrm{~mm}$ & & \\
\hline multiformin A & $\mathrm{ZI}=14 \mathrm{~mm}$ & \multirow[t]{4}{*}{ Hypoxylon multiforme (Fr.) Fr. (stromata) } & \multirow[t]{4}{*}{ [25] } \\
\hline multiformin B & $\mathrm{ZI}=19 \mathrm{~mm}$ & & \\
\hline multiformin C & $\mathrm{ZI}=18 \mathrm{~mm}$ & & \\
\hline multiformin D & $\mathrm{ZI}=17 \mathrm{~mm}$ & & \\
\hline \multicolumn{4}{|l|}{ Lactone derivatives } \\
\hline coloratin A & $\mathrm{ZI}=16 \mathrm{~mm}$ & $\begin{array}{l}\text { Xylaria intracolorata (J. D. Rogers, Callan \& Samuels) } \\
\text { J. D. Rogers \& Y. M. Ju (stromata) }\end{array}$ & [76] \\
\hline Coprinuslactone & $\mathrm{MIC}=150 \mu \mathrm{g} / \mathrm{mL}$ & Coprinus comatus (O. F. Müll.) Pers. (mycelium) & [33] \\
\hline \multicolumn{4}{|l|}{ Cyclohexenyl derivative } \\
\hline lentinoid B & $\mathrm{MIC}=160 \mu \mathrm{g} / \mathrm{mL}$ & Lentinus strigellus Berk. (mycelium) & {$[80]$} \\
\hline \multicolumn{4}{|l|}{ Phenolic acids } \\
\hline cinnamic acid & $\mathrm{MIC}=0.7 \mu \mathrm{g} / \mathrm{mL}$ & \multirow[t]{2}{*}{ Ganoderma lucidum (Curtis) P. Karst. (fruiting body) } & \multirow[t]{2}{*}{35} \\
\hline p-hydroxybenzoic acid & $\mathrm{MIC}=3 \mu \mathrm{g} / \mathrm{mL}$ & & \\
\hline \multicolumn{4}{|l|}{ Steroid } \\
\hline Ergosterol & $\begin{array}{l}\mathrm{ZI}=18 \mathrm{~mm} \\
\mathrm{MIC}=200 \mu \mathrm{g} / \mathrm{mL} \\
\mathrm{MBC}=250 \mu \mathrm{g} / \mathrm{mL}\end{array}$ & $\begin{array}{l}\text { Morchella esculenta (L.) Pers./Verpa bohemica } \\
\text { (Krombh.) J. Schröt. (fruiting body) }\end{array}$ & [37] \\
\hline \multicolumn{4}{|l|}{ Alkaloid } \\
\hline 2-aminoquinoline & $\mathrm{MIC}=128 \mu \mathrm{g} / \mathrm{mL}$ & Leucopaxillus albissimus (Peck) Singer (fruiting body) & [79] \\
\hline \multicolumn{4}{|l|}{ Chlorinated derivatives } \\
\hline drosophilin A & $\mathrm{MIC}=250 \mu \mathrm{g} / \mathrm{mL}$ & Drosophila subatrata (Batsch) Quél. (mycelium) & [50] \\
\hline gymnopalyne A & $\mathrm{MIC}=67 \mu \mathrm{g} / \mathrm{mL}$ & Gymnopus sp. (mycelium) & [71] \\
\hline \multicolumn{4}{|l|}{ Polyacetylene } \\
\hline drosophilin D & $\mathrm{MIC}=250 \mu \mathrm{g} / \mathrm{mL}$ & Drosophila subatrata (Batsch) Quél. (mycelium) & {$[50]$} \\
\hline
\end{tabular}

- Table 6 Compounds isolated from mushrooms with antibacterial activity against Enterobacter spp.

\begin{tabular}{|c|c|c|c|c|}
\hline $\begin{array}{l}\text { Chemical class } \\
\text { Compound }\end{array}$ & Inhibition & Bacterial species & Source & Ref \\
\hline \multicolumn{5}{|l|}{ Terphenyl quinone } \\
\hline atromentin & $50 \leq \mathrm{MIC} \leq 100 \mu \mathrm{g} / \mathrm{mL}$ & E. aerogenes ${ }^{\mathrm{a}}$ & Hydnaceae sp. (fruiting body) & [28] \\
\hline \multicolumn{5}{|l|}{ Phenolic acids } \\
\hline cinnamic acid & $\mathrm{MIC}=1.5 \mu \mathrm{g} / \mathrm{mL}$ & \multirow[t]{2}{*}{ E. cloacae } & \multirow[t]{2}{*}{ Ganoderma lucidum (Curtis) P. Karst. (fruiting body) } & \multirow[t]{2}{*}{ [35] } \\
\hline p-hydroxy-benzoic acid & $\mathrm{MIC}=6 \mu \mathrm{g} / \mathrm{mL}$ & & & \\
\hline
\end{tabular}


- Table 7 Compounds isolated from mushrooms with antibacterial activity against Escherichia coli.

\begin{tabular}{|c|c|c|c|}
\hline $\begin{array}{l}\text { Chemical class } \\
\text { Compound }\end{array}$ & Inhibition & Source & Ref \\
\hline \multicolumn{4}{|l|}{ Pyranoquinones } \\
\hline multiformin B & $\mathrm{ZI}=19 \mathrm{~mm}$ & \multirow[t]{3}{*}{ Hypoxylon multiforme (Fr.) Fr. (stromata) } & \multirow[t]{3}{*}{ [25] } \\
\hline multiformin C & $\mathrm{ZI}=18 \mathrm{~mm}$ & & \\
\hline multiformin D & $\mathrm{ZI}=15 \mathrm{~mm}$ & & \\
\hline sassafrin A & $\mathrm{ZI}=20 \mathrm{~mm}$ & \multirow{4}{*}{$\begin{array}{l}\text { Creosphaeria sassafras (Schwein.) Y. M. Ju, } \\
\text { F. San Martín \&J. D. Rogers (stromata) }\end{array}$} & \multirow[t]{4}{*}{ [24] } \\
\hline sassafrin B & $\mathrm{ZI}=14 \mathrm{~mm}$ & & \\
\hline sassafrin C & $\mathrm{ZI}=22 \mathrm{~mm}$ & & \\
\hline sassafrin D & $\mathrm{ZI}=19 \mathrm{~mm}$ & & \\
\hline \multicolumn{4}{|l|}{ Terphenyl quinones } \\
\hline spiromentin B & $\mathrm{MIC}=100 \mu \mathrm{g} / \mathrm{mL}^{\mathrm{a}}$ & \multirow[t]{2}{*}{ Tapinella atrotomentosa (Batsch) Šutara (fruiting body) } & \multirow[t]{2}{*}{ [27] } \\
\hline spiromentin C & $\mathrm{MIC}=10 \mu \mathrm{g} / \mathrm{mL}^{\mathrm{a}}$ & & \\
\hline atromentin & $50 \leq \mathrm{MIC} \leq 100 \mu \mathrm{g} / \mathrm{mL}$ & Hydnaceae sp. (fruiting body) & [28] \\
\hline \multicolumn{4}{|l|}{ Indole-quinone polymer } \\
\hline \multirow[t]{2}{*}{ melanin } & $\mathrm{ZI}=10.3 \mathrm{~mm}$ with $10 \mu \mathrm{g}$ & Armillaria mellea (Vahl) P. Kumm. (rhizomorph) & [30] \\
\hline & $\mathrm{MIC}=160 \mu \mathrm{g} / \mathrm{mL}$ & Auricularia auricula-judae (Bull.) Quél. (fruiting body) & [31] \\
\hline \multicolumn{4}{|l|}{ Lactone derivatives } \\
\hline 5-hydroxy-hex-2-en-4-olide & $\mathrm{MIC}=10 \mu \mathrm{g} / \mathrm{mL}^{\mathrm{a}}$ & \multirow[t]{2}{*}{ Tapinella atrotomentosa (Batsch) Šutara (fruiting body) } & \multirow[t]{2}{*}{ [27] } \\
\hline osmundalactone & $\mathrm{MIC}=10 \mu \mathrm{g} / \mathrm{mL}^{\mathrm{a}}$ & & \\
\hline coloratin A & $\mathrm{ZI}=16 \mathrm{~mm}$ & $\begin{array}{l}\text { Xylaria intracolorata (J. D. Rogers, Callan \& Samuels) } \\
\text { J.D. Rogers \& Y. M. Ju (stromata) }\end{array}$ & [76] \\
\hline \multicolumn{4}{|l|}{ Ketone derivatives } \\
\hline 2-octanone & $\mathrm{MIC}=125 \mu \mathrm{g} / \mathrm{g}$ & \multirow[t]{2}{*}{ Pleurotus ostreatus (Jacq.) P. Kumm. (fruiting body) } & \multirow[t]{2}{*}{ [34] } \\
\hline 3-octanone & $\mathrm{MIC}=125 \mu \mathrm{g} / \mathrm{g}$ & & \\
\hline \multicolumn{4}{|l|}{ Phenolic acids } \\
\hline cinnamic acid & $\mathrm{MIC}=7 \mu \mathrm{g} / \mathrm{mL}$ & \multirow[t]{2}{*}{ Ganoderma lucidum (Curtis) P. Karst. (fruiting body) } & \multirow[t]{2}{*}{ [35] } \\
\hline p-hydroxybenzoic acid & $\mathrm{MIC}=30 \mu \mathrm{g} / \mathrm{mL}$ & & \\
\hline \multicolumn{4}{|l|}{ Sesquiterpenoids } \\
\hline creolophin E & $\mathrm{MIC}=5 \mu \mathrm{g} / \mathrm{mL}$ & Creolophus cirrhatus (Pers.) P. Karst. (mycelium) & [41] \\
\hline phellodonic acid & $\mathrm{MIC}=100 \mu \mathrm{g} / \mathrm{mL}$ & Phellodon melaleucus (Sw. ex Fr.) P. Karst. (mycelium) & [39] \\
\hline stereumamide A & $\mathrm{MIC}=12.5 \mu \mathrm{g} / \mathrm{mL}$ & \multirow[t]{3}{*}{ Stereum hirsutum (Willd.) Pers. (mycelium) } & \multirow[t]{3}{*}{ [38] } \\
\hline stereumamide D & $\mathrm{MIC}=12.5 \mu \mathrm{g} / \mathrm{mL}$ & & \\
\hline sterostrein Q & $\mathrm{MIC}=12.5 \mu \mathrm{g} / \mathrm{mL}$ & & \\
\hline \multicolumn{4}{|l|}{ Diterpenoids } \\
\hline pleuromutilin & $\mathrm{MIC}=250 \mu \mathrm{g} / \mathrm{mL}$ & Pleurotus passeckerianus Pilát (mycelium) & [50] \\
\hline cyathinin A & $\mathrm{I} \mathrm{C}_{50}=25 \mu \mathrm{g} / \mathrm{mL}^{\mathrm{b}}$ & \multirow{6}{*}{$\begin{array}{l}\text { Cyathus subglobisporus R. L. Zhao, Desjardin \& K. D. } \\
\text { Hyde (mycelium) }\end{array}$} & \multirow[t]{6}{*}{ [43] } \\
\hline cyathinin D & $\mathrm{IC}_{50}=12.5 \mu \mathrm{g} / \mathrm{mL}^{\mathrm{b}}$ & & \\
\hline striatal A & $I C_{50}=6.25 \mu \mathrm{g} / \mathrm{mL}^{\mathrm{b}}$ & & \\
\hline striatal B & $\mathrm{IC}_{50}=3.13 \mu \mathrm{g} / \mathrm{mL}^{\mathrm{b}}$ & & \\
\hline striatal D & $\mathrm{IC}_{50}=50 \mu \mathrm{g} / \mathrm{mL}^{\mathrm{b}}$ & & \\
\hline striatoid C & $\mathrm{IC}_{50}=50 \mu \mathrm{g} / \mathrm{mL}^{\mathrm{b}}$ & & \\
\hline \multicolumn{4}{|l|}{ Steroids } \\
\hline ergosterol & $\begin{array}{l}\mathrm{ZI}=21 \mathrm{~mm} \\
\mathrm{MIC}=300 \mu \mathrm{g} / \mathrm{mL}\end{array}$ & $\begin{array}{l}\text { Morchella esculenta (L.) Pers./Verpa bohemica } \\
\text { (Krombh.) J. Schröt. (fruiting body) }\end{array}$ & $\begin{array}{l}{[37]} \\
\text { cont. }\end{array}$ \\
\hline
\end{tabular}


- Table 7 Continued

\begin{tabular}{|c|c|c|c|}
\hline $\begin{array}{l}\text { Chemical class } \\
\text { Compound }\end{array}$ & Inhibition & Source & Ref \\
\hline \multicolumn{4}{|l|}{ Alkaloids (piperazine derivatives) } \\
\hline emestrin & $\mathrm{IC}_{50}=2.21 \mu \mathrm{g} / \mathrm{mL}$ & $\begin{array}{l}\text { Armillaria tabescens (Scop.) R. A. Koch \& Aime } \\
\text { (mycelium) }\end{array}$ & [55] \\
\hline epicorazine A & $\mathrm{ZI}=25 \mathrm{~mm}$ with $100 \mu \mathrm{g}$ & \multirow[t]{3}{*}{ Podaxis pistillaris (L.) Fr. (mycelium) } & \multirow[t]{3}{*}[54]{} \\
\hline epicorazine B & $\mathrm{ZI}=24 \mathrm{~mm}$ with $100 \mu \mathrm{g}$ & & \\
\hline epicorazine C & $\mathrm{ZI}=18 \mathrm{~mm}$ with $100 \mu \mathrm{g}$ & & \\
\hline \multicolumn{4}{|l|}{ Sulfur derivative } \\
\hline bis((methylsulfonyl) methyl)disulfide & $\mathrm{MIC}=100 \mu \mathrm{g} / \mathrm{mL}$ & Lentinus edodes (Berk.) Singer (fruiting body) & {$[73]$} \\
\hline \multicolumn{4}{|l|}{ Chlorinated derivatives } \\
\hline drosophilin A & $\mathrm{MIC}=250 \mu \mathrm{g} / \mathrm{mL}$ & Drosophila subatrata (Batsch) Quél. (mycelium) & {$[50]$} \\
\hline tetrachloropyro-catechol & $\mathrm{MIC}=20 \mu \mathrm{g} / \mathrm{mL}$ & \multirow[t]{2}{*}{ Mycena sp. (mycelium) } & \multirow[t]{2}{*}[72]{} \\
\hline tetrachloropyro-catechol methylether & $\mathrm{MIC}=50 \mu \mathrm{g} / \mathrm{mL}$ & & \\
\hline \multicolumn{4}{|l|}{ Alcohols } \\
\hline octan-1-ol & $\mathrm{MIC}=125 \mu \mathrm{g} / \mathrm{g}$ & \multirow[t]{2}{*}{ Pleurotus ostreatus (Jacq.) P. Kumm. (fruiting body) } & \multirow[t]{2}{*}[34]{} \\
\hline octan-3-ol & $\mathrm{MIC}=125 \mu \mathrm{g} / \mathrm{g}$ & & \\
\hline \multicolumn{4}{|l|}{ Fatty acid } \\
\hline pentadecanoic acid & $\begin{array}{l}\mathrm{ZI}=21 \mathrm{~mm} \\
\mathrm{MIC}=250 \mu \mathrm{g} / \mathrm{mL}\end{array}$ & $\begin{array}{l}\text { Morchella esculenta (L.) Pers./Verpa bohemica } \\
\text { (Krombh.) J. Schröt. (fruiting body) }\end{array}$ & {$[37]$} \\
\hline \multicolumn{4}{|l|}{ Polyacetylene } \\
\hline drosophilin D & $\mathrm{MIC}=250 \mu \mathrm{g} / \mathrm{mL}$ & Drosophila subatrata (Batsch) Quél. (mycelium) & {$[50]$} \\
\hline \multicolumn{4}{|l|}{ Polysaccharides } \\
\hline polysaccharides & $\mathrm{MIC}=12.5 \mu \mathrm{g} / \mathrm{mL}$ & $\begin{array}{l}\text { Lentinus edodes (Berk.) Singer (spent mushroom } \\
\text { substrate) }\end{array}$ & {$[66]$} \\
\hline exopolysaccharides & $\mathrm{ZI}=15 \mathrm{~mm}$ & Lentinus edodes (Berk.) Singer (mycelium) & {$[65]$} \\
\hline \multicolumn{4}{|l|}{ Protein } \\
\hline Cordyceps sinensis antibacterial protein & $\mathrm{MIC}=75-100 \mu \mathrm{g} / \mathrm{mL}$ & Cordyceps sinensis (Berk.) Sacc. (mycelium) & {$[62]$} \\
\hline
\end{tabular}

the data regarding bioactivity of mushroom extracts and metabolites against $A$. baumannii are rare. In addition, spiromentin $C$ also inhibits methicillin-resistant Staphylococcus aureus (MRSA) growth to a lesser extent $(\mathrm{MIC}=250 \mu \mathrm{g} / \mathrm{mL})$, and its analogue spiromentin $B$ also affects the growth of ESBL E. coli. These compounds have been also investigated in combination with cefuroxime against MRSA, but no synergistic effect has been observed. Yet, further toxicological tests are warranted to guarantee the safety of these compounds for human cells, to confirm therefore their potential interest as future antibiotics. Another terphenyl quinone from several Hydnaceae species, atromentin, has shown antibacterial activity against $E$. coli and Enterobacter aerogenes (now included in the Klebsiella genus), a nosocomial bacteria leading to opportunistic infections, and known to acquire resistance mechanisms [28]. Even if MIC values are quite high $(50-100 \mu \mathrm{g} / \mathrm{mL})$ compared to penicillins against clinical strains, this result remains quite promising. Atromentin has been reported to inhibit the enoyl-acyl carrier protein (ACP) reductase of S. pneumoniae, an enzyme essential for the biosynthesis of fatty acids, but without any effect on the enoyl-ACP reductase of S. aureus or E. coli. There- fore, its mode of action explaining the inhibition of $E$. coli and $E$. aerogenes still has to be elucidated [29].

Melanins are particular quinone derivatives, commonly described as high molecular weight insoluble pigments, derived from the oxidation of phenols leading to heterogeneous indolequinone polymers. Although fungal melanin structures are still not well characterized, they have been studied for biological activities. Raw melanin isolated from Armillaria melea (Agaricale, Basidiomycetes) shows antibacterial activities against $P$. aeruginosa, and, to a less extent, toward S. aureus and E. coli [30]. However, once purified, its inhibitory activity against $P$. aeruginosa decreases, and disappears completely with regard to $S$. aureus and E. coli. This could be due to the linkage of melanin with antimicrobial polysaccharides in the raw fraction. However, melanin from the jelly ear mushroom (Auricularia auricula-judae) has been reported to inhibit the growth of $E$. coli and $P$. aeruginosa, with MIC values of $160 \mu \mathrm{g} / \mathrm{mL}$ and $640 \mu \mathrm{g} / \mathrm{mL}$, respectively. In addition, it was also found to inhibit biofilm formation at values below MIC [31]. This antibiofilm effect is regulated by quorum sensing and does not interfere with bacteria growth. Authors suggest that 
<smiles>COc1cc(O)c2c(c1)C(=O)c1cc(C)cc(O)c1C2=O</smiles>

1<smiles>CCC(C)/C=C(C)/C=C/C(=O)/C=C(\O)C1=C2C3=CC(=O)O[C@@](C)(C(CC(C)O)=C3)C2=CC(=O)C1</smiles>

2

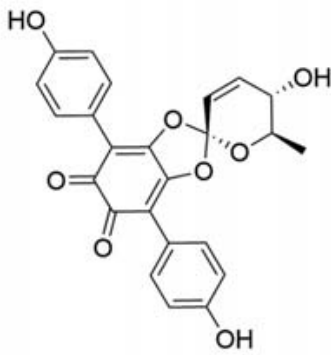

3<smiles>C=C1C(=O)O[C@@H](C)[C@H]1O</smiles>

5<smiles>CC(O)[C@H](C(=O)[O-])[n+]1ccc2c(c1)C(=O)C1=CC(C)(C)C[C@H]1[C@H]2O</smiles>

6

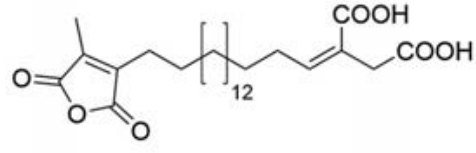

4

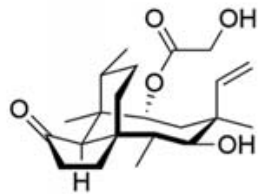

8<smiles>COc1c(Cl)c(Cl)c(O)c(Cl)c1Cl</smiles>

10

\section{Lactone, Ketone, and Phenolic Derivatives}

Thirteen lactones from a polypore species of Skeletocutis (Basidiomycetes) have been tested for their activity against methicillinsensitive and -resistant S. aureus: skeletocutins $\mathrm{K}(4)$ and $\mathrm{L}$ show the best antibacterial effect against both strains [32]. Interestingly, skeletocutin I inhibits the biofilm formation of $S$. aureus but has no significant effect on bacterial growth (the antibiofilm activity of skeletocutins $\mathrm{K}$ and $\mathrm{L}$ has not been evaluated in this study). Furthermore, these lactone derivatives did not show any cytotoxicity against mouse fibroblast and HeLa cell lines when tested at concentrations of $37 \mu \mathrm{g} / \mathrm{mL}$, warranting further investigations. Two lactones isolated from the velvet roll-rim also possess interesting antibacterial activities: they strongly inhibit multiresistant $A$. bau-

mannii and ESBL-producing E. coli, with $\mathrm{MIC} \leq 10 \mu \mathrm{g} / \mathrm{mL}$, and have also an effect on MRSA [27]. Nevertheless, cytotoxicity and stability assessments should be carried out prior to considering these molecules as potential drug-candidates. Activity-directed fractionation of Coprinus comatus (Psathyrellaceae) led to the isolation of coprinuslactone (5), which shows moderate bacteriostatic and antibacterial effects against $P$. aeruginosa and $S$. aureus. This lactone also exerts a bactericidal activity at twice its MIC value and dissolves in vitro-established biofilms of $P$. aeruginosa and $S$. aureus at concentrations below MIC [33]. Coprinuslactone has shown interesting quorum quenching activity against $E$. coli at subtoxic concentration, which could explain its ability to disrupt bacterial biofilm; less than $4 \mu \mathrm{g} / \mathrm{mL}$ was sufficient to completely abolish the quorum sensing response of a specific autoinducer. Coprinulactone also interferes with peptidoglycan synthesis by binding to the MurA enzyme and inhibiting it. Moreover, observation of the structure of the cell membrane after treatment with this compound suggests that it can destabilize the latter. Finally, this lactone has showed weak cytostatic but no cytotoxic effect against a mouse fibroblast cell line [33]. Lactones are often reactive molecules, with the risk of ring-opening and loss of efficacy; however, all these lactones (skeletocutins, coprinulactone, 5-hydroxy-hex2-en-4-olide, and osmundalactone) are $\gamma$ - or $\delta$-lactones, which 
could be stable enough to be considered for further investigations regarding their antibiotic potential.

Some volatile ketones and their corresponding alcohols have been isolated from the edible oyster mushroom Pleurotus ostreatus. A mixture of these compounds in concentrations imitating the composition of fruiting bodies of $P$. ostreatus has inhibited the growth of all tested bacterial strains, including $K$. pneumoniae and $P$. aeruginosa [34]. However, none of these compounds displays remarkable antibacterial effects, and drug development is difficult with volatile compounds, limiting thereby the potential of these molecules.

With regard to phenolic compounds, no molecule specific to the fungi kingdom has been reported to exert antibacterial effects toward ESKAPEE bacteria. The ubiquitous cinnamic and p-hydroxybenzoic acids (isolated from Ganoderma lucidum) were evaluated for antibacterial activity on S. aureus, P. aeruginosa, E. coli, and Enterobacter cloacae, and they showed significant effects with MIC values comprised between 0.7 and $30 \mu \mathrm{g} / \mathrm{mL}$ [35]. Based on literature suggesting that phenolic compounds are rapidly metabolized and mostly glucuronidated after human consumption, authors produced acetylated glucuronide derivatives from the parent compounds: these derivatives showed a still high antibacterial activity but nevertheless lower than that of the original phenolic acids.

\section{Terpenoids}

Antibacterial terpenoids identified in mushrooms are mainly sesqui- and diterpene derivatives.

Regarding larger compounds, only ganoderic acid has been identified as a weak antibacterial triterpene against $S$. aureus but leading to a strong cytotoxicity toward HeLa and U87 glioma cancer cells [36]. The ubiquitous ergosterol (isolated from morel mushrooms) is also the only steroid reported to be active toward pathogenic bacteria, with a minimal bactericidal concentration of $250 \mu \mathrm{g} / \mathrm{mL}$ against $P$. aeruginosa, twice less active against $E$. coli [37].

Regarding sesquiterpenoids, 9 fungal compounds have been reported to inhibit the growth of $S$. aureus or $E$. coli. Stereumamides $A(6)$ and $D$, resulting from the conjugation of sesquiterpenes with an $\alpha$-amino acid, were found to have a similar activity on S. aureus and E. coli growth (MIC $=12.5 \mu \mathrm{g} / \mathrm{mL}$ ), while sterostrein $\mathrm{Q}$ was slightly less active on $S$. aureus than against $E$. coli [38]. Phellodonic acid, an hirsutane-type sesquiterpene, has also shown a potent antibacterial activity against $S$. aureus $(\mathrm{MIC}=$ $10 \mu \mathrm{g} / \mathrm{mL}$ ), less against $E$. coli with a 10-times higher MIC value [39]. However, phellodonic acid is toxic toward several mammalian cells lines, including HeLa human cells ( $\mathrm{IC}_{50}$ of $\left.10 \mu \mathrm{g} / \mathrm{mL}\right)$, thus preventing its development without appropriate structural modifications. Among other active sesquiterpenoids are found enokipodins $A-D$, cuparene derivatives isolated from the mushroom Flammulina velutipes (Physalacriaceae, Basidiomycetes) [40]. Enokipodins $A$ and $C$ show a higher antibacterial activity against S. aureus ( $\mathrm{ZI}$ of 25.2 and $21 \mathrm{~mm}$, respectively) compared to enokipodins $B$ and $D$, which are respective benzoquinone-type derivatives resulting from the oxidation of enokipodins $A$ and $C$. However, none of these compounds inhibited the growth of Gram- negative bacteria such as E. coli. On the contrary, creolophin E, a norhirsutane sesquiterpene from the rare Creolophus cirrhatus, inhibits the development of $E$. coli with a minimal inhibitory concentration of $5 \mu \mathrm{g} / \mathrm{mL}$ [41]. Unfortunately, as for phellodonic acid, this compound is also strongly cytotoxic toward various human cell lines $\left(\mathrm{IC}_{50} \leq 7 \mu \mathrm{g} / \mathrm{mL}\right)$. Ganomycins $A$ and B from Ganoderma pfeifferi are meroterpenoids exhibiting a farnesyl hydroquinone structure; they were evaluated both by diffusion and microdilution assay on 4 different strains of $S$. aureus, as well as on P. aeruginosa and E. coli, with ampicillin used as a positive control. Both ganomycins inhibit S. aureus growth with an MIC of $25 \mu \mathrm{g} / \mathrm{mL}$, but no evaluation of toxicity toward eukaryotic cells has been carried out [42].

Several mushroom diterpenoids have also been identified as significant antibacterial compounds. An interesting publication reports the evaluation of cyathane diterpenes isolated from Cyathus subglobisporus toward several ESKAPEE bacteria, including a multi-resistant $E$. faecium strain, an ESBL-producing $K$. pneumoniae, and the other Gram-negative E. coli, P. aeruginosa, and A. baumannii [43]. Evaluations have been performed according to CLSI guidelines, and in the presence of $\mathrm{PA} \beta \mathrm{N}$, a pump efflux inhibitor, in the case of Gram-negative bacteria. All compounds showed various antibacterial activities. Striatal A (7) was found to inhibit significantly E. faecium, K. pneumoniae, A. baumannii, and E. coli growth, with $\mathrm{IC}_{50}$ comprised between 6 and $50 \mu \mathrm{g} / \mathrm{mL}$. Striatal B also strongly inhibited the growth of $E$. faecium and A. baumannii (with $\mathrm{PA} \beta \mathrm{N}$ ), while striatin $\mathrm{C}$ and striatal $C$ were very active against $A$. baumannii and $E$. coli, respectively, in the presence of PA $\beta \mathrm{N}$. However, all the active compounds also displayed cytotoxicity against various cancerous and noncancerous cells with $\mathrm{IC}_{50}$ values in the $\mu \mathrm{M}$ range, which nuances the interest for these molecules as potential antibiotics. Two other cyathane derivatives isolated from the edible mushroom Strobilurus ohshimae have also shown weak bioactivity against $P$. aeruginosa but again are associated with cytotoxicity toward cancer cells [44]. Also with respect to cyathane derivatives, sarcodonins $M$ and $L$ displayed antibacterial activity against $S$. aureus in a disk diffusion assay, where disks of $6 \mathrm{~mm}$ of diameter were soaked with increasing concentrations of test compounds; no evaluation on mammalian cells has been reported for these molecules [45].

Several diterpenoids isolated from cultures of the basidiomycete Psathyrella candolleana possess antibacterial properties against Gram-positive bacteria. Psathyrelloic acid inhibits $S$. aureus but has no effect on P. aeruginosa and E. coli [46]. Similarly, psathyrins A and B, 2 tetracyclic diterpenoids, also inhibit weakly the growth of $S$. aureus without any effect toward $P$. aeruginosa [47]. While the first publication reports an MIC value, the second one mentions $\mathrm{MIC}_{50}$. While an MIC value is determined for a given isolate, the $\mathrm{MIC}_{50}$ corresponds to the lowest drug concentration that inhibit $50 \%$ of a large panel of isolates of a given species, namely S. aureus in this study. It seems that only one ATCC strain has been evaluated in this work; therefore, the data should be considered as MIC and not $\mathrm{MIC}_{50}$. None of these compounds from $P$. candolleana have been submitted to cytotoxic assay to evaluate their potential safety for human cells.

Another important antibacterial diterpene is pleuromutilin (8). This compound, firstly isolated in 1951 from Pleurotus mutilus 
(now renamed Clitopilus scyphoides), has also been identified in other Agaricales species, mostly from the genus Clitopilus as well as from the closely-related Omphalina mutila $[48,49]$. Pleuromutilin shows high antibacterial activity against $S$. aureus and $K$. pneumoniae (MIC of 0.25 and $1 \mu \mathrm{g} / \mathrm{mL}$, respectively), while its activity against $E$. coli and $P$. aeruginosa is very weak $[48,50]$. Also, pleuromutilin is not toxic to mice at a dose of $50 \mathrm{mg} / \mathrm{kg}$ of body weight, evaluated by intravenous and intraperitoneal route. Semi-synthetic derivatives of pleuromutilin have been further developed and form now the antibiotic class of pleuromutilins [51]. Initially used in veterinary medicine, some pleuromutilins have been approved for human medicine; for example, retapamulin is used as a topical agent for the treatment of impetigo or infected wounds caused by S. aureus, and more recently, lefamulin has been approved to treat some community-acquired bacterial pneumonia [8]. Other indications, including pediatric use, are currently undergoing phase I trials. Pleuromutilins inhibit bacterial proteins synthesis by binding to the $50 \mathrm{~S}$ ribosomal subunit and thus inhibit the formation of peptide bonds. Due to this unique interaction with the bacterial ribosome, there is no cross-resistance with current antibiotics targeting the biosynthesis of proteins, like tetracyclines or macrolides [52]. Pleuromutilins show a wide spectrum of action against $S$. aureus and $E$. faecium, including resistant strains, and recent "extended spectrum" pleuromutilins display an interesting activity against Enterobacteriaceae, including carbapenem-resistant strains. Recent investigations aim to further extend the antibacterial spectrum to include more ESKAPE pathogens. During its clinical evaluation against community-acquired bacterial pneumonia, lefamulin was globally well tolerated by patients, with the most frequent side effect being diarrhea, often observed with all antibiotics, even though studies have shown that lefamulin has a rather low impact on the intestinal microbiome [51,53]. Similarly, some vaginal fungal infections can also appear. No genotoxicity has been reported, but lefamulin has induced some teratogenic effects on rodent fetus in vivo; therefore, it should not be used by pregnant and breastfeeding patients, and women of childbearing potential should use effective contraception during treatment. In addition, it has to be noted that lefamulin is primarily metabolized by CYP3A4; hence it can interact with many other drugs. Up to now, pleuromutilins represent the only class of marketed antibiotics derived from macromycetes.

\section{Alkaloids}

Certain macrofungi alkaloids have interesting antibacterial activities against ESKAPEE bacteria. Epicorazines A-C from Podaxis pistillaris (Podaxaceae) are piperazine alkaloids containing a disulfide bridge. They inhibit the growth of S. aureus in a disk diffusion assay, as well as the one of E. coli, to a lesser extent [54]. It seems that the presence of a hydrogen on the beta position of $C 6^{\prime}$ as well as the double bond between $\mathrm{C}^{\prime}$ and $\mathrm{C}^{\prime}{ }^{\prime}$ increase their antibacterial activity. However, all of these compounds also show cytotoxic activities against human cells at antibacterial concentrations, which is a break for further development. Another antibacterial piperazine alkaloid isolated from the plant pathogen Armillaria tabescens, emestrin (9), exerts a remarkable inhibition activity against $E$. coli $\left(\mathrm{IC}_{50}=2.21 \mu \mathrm{g} / \mathrm{mL}\right)$ as well as against both sensitive and methicillin-resistant S. aureus [55]. Emestrin has also been reported to have antifungal properties, but no toxicological test toward human cell lines has been carried out to our knowledge. Cytochalasins are fungal metabolites consisting of a substituted isoindole scaffold fused with a macrocyclic ring. Several of these compounds, isolated from macromycetes but also from micromycetes, have been evaluated on $S$. aureus growth and biofilm production. Though they have not any significant effect on bacterial growth, some of them were found to strongly interfere with biofilm formation (up to $90 \%$ of inhibition when tested at $256 \mu \mathrm{g} / \mathrm{mL}$ ) [56]. Cytochalasan derivatives are therefore supposed to act on the quorum sensing of $S$. aureus, but the mechanism of action remains to be elucidated. Nevertheless, these compounds are known to be toxic agents, binding to actin filaments and blocking their polymerization, thus limiting their use in humans.

\section{Peptides and Proteins}

Beside small secondary metabolites, some polypeptides and proteins with antibacterial activity have also been identified from macromycetes, referred to as fungal defensins. The most interesting one is plectasin, a peptide composed of 40 amino acids and isolated from the ebony cup fungi, Pseudoplectania nigrella (Psathyrellaceae, Basidiomycetes). Plectasin shows interesting activity against both $S$. aureus and E. faecium; according to the strains tested (including clinical isolates, MRSA, and vancomycin-resistant $E$. faecium), MIC values range from $4 \mu \mathrm{g} / \mathrm{mL}$ to $128 \mu \mathrm{g} / \mathrm{mL}$. Activity results from the ability of plectasin to bind directly to lipid II, the essential precursor of the cell wall $[57,58]$. Furthermore, no cytotoxicity has been reported against human epidermal keratinocytes and murine fibroblasts. In 2008, a derivative of plectasin, NZ2114, developed by Novozymes and Sanofi-Aventis, is more efficient and less toxic than the original peptide, and active against a broad range of Gram-positive bacterial strains resistant to existing antibiotics [59]. There are numerous recent studies dealing with bioassays, optimization, and large-scale production of NZ2114 and other plectasin derivatives, highlighting the promising potential of these fungal defensins. Another fungal defensin with the same mode of action, copsin, was identified from Coprinopsis cinerea (Sarcosomataceae, Ascomycetes). This 57 amino acids peptide shows high bactericidal activity against $E$. faecium $(\mathrm{MBC}=$ $4 \mu \mathrm{g} / \mathrm{mL}$ ), even more on a vancomycin-resistant strain $(\mathrm{MBC}=$ $2 \mu \mathrm{g} / \mathrm{mL}$ ), but has no effect on $S$. aureus at the maximum tested concentration of $64 \mu \mathrm{g} / \mathrm{mL}$ [60]. The cytotoxicity of copsin has not been investigated yet. Some peptaibols from boletus also inhibit the growth of sensitive and methicillin-resistant S. aureus in a disk diffusion assay [61].

Besides those polypeptides, some proteins with antibacterial effects have also been identified from macromycetes. The Cordyceps sinensis antibacterial protein (CSAP) isolated from Cordyceps sinensis (now renamed Ophiocordyceps sinensis, Ascomycetes) exhibits a moderate inhibitory activity on the growth of $E$. coli and S. aureus [62], as well as F2 protein from the common mushroom Agaricus bisporus (Agaricaceae), inhibiting the growth of both methicillin-sensitive and resistant S. aureus [63]. A ribonuclease isolated from the edible mushroom Lentinus sajor-caju (formerly known as Pleurotus sajor-caju) was also found to inhibit the 
growth of $S$. aureus and $P$. aeruginosa, but its further usage as antibiotic is excluded due its antimitogenic effects on normal and cancer cell lines [64].

\section{Polysaccharides}

Several polysaccharides from macromycetes have shown activity against ESKAPEE bacteria, including polysaccharides isolated from Lentinus edodes and from their spent substrate. These polysaccharides, not structurally described, have displayed some activity against $K$. pneumoniae and E. coli $[65,66]$. Several other publications report antibacterial mushroom polysaccharides but generally with MIC values in the $\mathrm{mg} / \mathrm{mL}$ range [67-69], which is too weak to consider any further development. In addition, polysaccharides are complex compounds whose molecular structure is generally not always fully elucidated. Nevertheless, new formulation strategies can reinforce the antibacterial potential of fungal polysaccharides. For instance, silver nanoparticles with glucans from Pleurotus florida have been shown to be effective against a multiresistant strain of $K$. pneumoniae, with an MIC value of $40 \mu \mathrm{g} / \mathrm{mL}[70]$.

\section{Other Compounds}

Basidiomycetes are known for their capacity to produce organohalogen compounds, mostly chlorinated metabolites. These compounds exhibit various properties, including antibacterial effects. For instance, the polychlorinated phenol drosophilin A (10), isolated from Drosophila subatrata (now Parasola conopilus), displays significant antibacterial activity against $S$. aureus and K. pneumoniae (MIC comprised between 4 and $64 \mu \mathrm{g} / \mathrm{mL}$ ), while its inhibitory effect is more moderate on the growth of $P$. aeruginosa and E. coli (MIC $=250 \mu \mathrm{g} / \mathrm{mL}$ ) [50]. Gymnopalyne A, a chlorinated isocoumarin from the cultures of Gymnopus sp. (Basidiomycetes), inhibits $P$. aeruginosa, with an MIC value equivalent to gentamycin; this compound is quite selective as it does not affect $S$. aureus and E. coli growth [71]. Two other chlorinated compounds from Mycena species (Tricholomataceae), tetrachloropyrocatechol and its methylated derivative, exert antibacterial effects against $E$. coli (MIC values of 20 and $50 \mu \mathrm{g} / \mathrm{mL}$, respectively); substitution of a hydroxyl group by a methyl function seems to decrease the bioactivity [72].

Sulfur compounds are also particular metabolites occurring in mushrooms. One of them isolated from the edible mushroom Lentinus edodes shows high and moderate antibacterial effect against $S$. aureus and E. coli, respectively, but no significant activity against $P$. aeruginosa [73].

Pentadecanoic acid, a saturated fatty acid purified from wild morel species, displays a weak antibacterial activity against S. aureus and E. coli; it probably acts as a bactericidal agent as $\mathrm{MBC}$ are close to MIC values for these 2 bacterial strains [37].

Finally, a polyacetylene from Parasola conopilus, drosophilin D, shows high inhibitory effects on $S$. aureus, moderate against $P$. aeruginosa and E. coli, and insignificant on K. pneumoniae [50].

\section{Concluding Remarks and Perspectives}

Bacterial infections still represent a major health threat for all countries, even industrialized ones with access to modern therapies. In the same way that bacteria have adapted to our antibiotics, it is now our turn to adapt through the development of new drugs, to block the growing phenomenon of multidrug resistance.

Nature has proven to be a huge reservoir of drug candidates, and mushrooms could be an underexplored resource for novel antibacterial products with original scaffolds, as illustrated with pleuromutilins. However, one of the main obstacles encountered in the development of natural molecules is the supply of active ingredients, and mushrooms could be problematic in terms of biomass and overall yield. Some species can grow rapidly; otherwise, optimized cultivation under sterile conditions could be considered, as well as high-producing strains selection. Another option would be to identify the genes involved in the biosynthesis of the metabolite of interest and introduce them in the plasmid of a yeast cell, for example. A genetic engineering process is generally suitable to meet the industrial scale-up. This strategy has been successfully applied to produce pleuromutilin; the gene cluster was inserted within an Aspergillus strain, enabling a significant increase of pleuromutilin production (2106\%) [74]. For small compounds having a rather simple chemical structure-like coprinuslactone or drosophiline A-production by total chemical synthesis could be more attractive from an economic point of view. Finally, active compounds identified in mushrooms can be specific to the fungi kingdom but also more widespread like the anthraquinone parietin, occurring in some lichens or plants. In this case, isolation of the active compound could be carried out from plants if global yield, process, and cost are more profitable. It has also to be noted that macromycetes can host endophytic microorganisms with potential properties. For example, an endophytic Enterobacter species isolated from the common mushroom Agaricus bisporus was found to produce several antibacterial compounds [75]. The production of such molecules through the cultivation of endophytic organisms could be another interesting alternative.

Regarding bioactivity, one of our main expectations is that the chemical diversity of mushrooms could be associated with original modes of action, as illustrated with coprinulactone or plectasin. Very few of the publications mentioned in this review have gone so far as to study the mechanisms involved; however, this must be the subject of in-depth studies, whether for the direct effect on bacterial growth or for biofilm inhibition, which represents a promising area of investigation. Also, the characterization of these modes of action is crucial to anticipate the emergence of new resistance mechanisms toward active fungal metabolites. Finally, as already mentioned, cytotoxicity must be evaluated on eukaryotic cells before considering further development as antibiotics, as well as in vivo validation of efficacy and safety.

In conclusion, the information summarized in this review emphasizes the potentiality of some fungal compounds as alternative agents against pathogenic bacteria. Despite global awareness of the antibiotic crisis and intense research from the scientific community, new effective antibiotics are struggling to emerge. We are aware that the road leading to a novel drug-candidate is long and tedious. Nevertheless, we are convinced that mushrooms repre- 
sent a yet unexplored source of promising antibacterial compounds, which deserves to be investigated while being preserved.

\section{Supporting Information}

The antibacterial activities presented in this review (IC 50 or MIC) are also available in $\mu \mathrm{M}$ in Supporting Information.

\section{Contributors' Statement}

Conception and design: A. Urbain, M. Bourjot, C. Huguet; data collection and analysis: V. Hamers, A. Urbain, C. Huguet; draft manuscript: V. Hamers, A. Urbain, C. Huguet; critical review: A. Urbain, C. Huguet, M. Bourjot, V. Hamers.

\section{Acknowledgements}

Dr Emilie Blaise is gratefully acknowledged for proofreading and English language editing of this manuscript.

\section{Conflict of Interest}

The authors declare that they have no conflict of interest.

\section{References}

[1] Patridge E, Gareiss P, Kinch MS, Hoyer D. An analysis of FDA-approved drugs: natural products and their derivatives. Drug Discov Today 2016; 21: 204-207

[2] Zhou L, Zhao J, Xu L, Huang Y, Ma Z, Wang J, Jiang W. Antimicrobial Compounds produced by Plant endophytic Fungi. In: De Costa P, Bezerra $P$ eds. Fungicides: Chemistry, environmental Impact and Health Effects. New York: Nova Science Publishers; 2009: 91-119

[3] Habbu P, Warad V, Shastri R, Madagundi S, Kulkarni VH. Antimicrobial metabolites from marine microorganisms. Chin J Nat Med 2016; 14: 101-116

[4] Gargano ML, van Griensven LJLD, Isikhuemhen OS, Lindequist U, Venturella G, Wasser SP, Zervakis GI. Medicinal mushrooms: Valuable biological resources of high exploitation potential. Plant Biosyst 2017; 151: 548-565

[5] Chaturvedi VK, Agarwal S, Gupta KK, Ramteke PW, Singh MP. Medicinal mushroom: boon for therapeutic applications. 3 Biotech 2018; 8: 334354

[6] Schwan WR. Mushrooms: an untapped reservoir for nutraceutical antibacterial applications and antibacterial compounds. Curr Top Nutraceutical Res 2012; 10: 75-82

[7] Alves M], Ferreira ICFR, Dias ], Teixeira V, Martins A, Pintado M. A review on antimicrobial activity of mushroom (Basidiomycetes) extracts and isolated compounds. Planta Med 2012; 78: 1707-1718

[8] Veve MP, Wagner JL. Lefamulin: review of a promising novel pleuromutilin antibiotic. Pharmacotherapy 2018; 38: 935-946

[9] Mulani MS, Kamble EE, Kumkar SN, Tawre M, Pardesi K. Emerging strategies to combat ESKAPE pathogens in the era of antimicrobial resistance: a review. Front Microbiol 2019; 10: 539

[10] Santajit S, Indrawattana N. Mechanisms of antimicrobial resistance in ESKAPE pathogens. Biomed Res Int 2016; 2016: 2475067. doi:10.1155/ 2016/2475067

[11] Cassini A, Högberg LD, Plachouras D, Quattrochi A, Hoxha A, Simonsen GS, Colomb-Cotinat M, Kretzschmar ME, Devleesschauwer B, Cecchini M, Ouakrim DA, Oliveira TC, Struelens M, Suetens C, Monnet DL, Burden of AMR Collaborative Group. Attributable deaths and disability-adjusted life-years caused by infections with antibiotic-resistant bacteria in the EU and the European Economic Area in 2015: a population-level modelling analysis. Lancet Infect Dis 2019; 19: 56-66

[12] Centers for Disease Control and Prevention. Antibiotic Resistance Threats in the United States, 2019. Atlanta, GA: U.S. Department of Health and Human Services, CDC; 2019

[13] Interagency Coordination Group (IACG) on Antimicrobial Resistance. No Time to wait: Securing the Future from drug-resistant Infections. New York: United Nations; 2019

[14] Zhen X, Lundborg CS, Sun X, Hu X, Dong H. Economic burden of antibiotic resistance in ESKAPE organisms: a systematic review. Antimicrob Resist Infect Control 2019; 8: 137

[15] Touat M, Opatowski M, Brun-Buisson C, Cosker K, Guillemot D, Salomon J, Tuppin $\mathrm{P}$, Lagasner $\mathrm{G}$, Watier $\mathrm{L}$. A payer perspective of the hospital inpatient additional care costs of antimicrobial resistance in France: a matched case-control study. Appl Health Econ Health Policy 2019; 17 : 381-389

[16] Tacconelli E, Carrara E, Savoldi A, Harbarth S, Mendelson M, Monnet D, Pulcini C, Kahlmet G, Kluytmans J, Carmeli Y, Ouellette M, Outterson K, Patel J, Cavaleri M, Cox EM, Houchens C, Grayson M, Hansen P, Singh N, Theuretzbacher U, Magrini N, The WHO Pathogens Priority List Working Group. Discovery, research, and development of new antibiotics: the WHO priority list of antibiotic-resistant bacteria and tuberculosis. Lancet Infect Dis 2018; 18: 318-327

[17] Pendleton JN, Gorman SP, Gilmore BF. Clinical relevance of the ESKAPE pathogens. Expert Rev Anti Infect Ther 2013; 11: 297-308

[18] Kurhekar J, Tupas GD, Otero MCB. In-vitro Assays for antimicrobial Assessment. In: Kumar S, Egbuna C, eds. Phytochemistry: An in-silico and in-vitro Update: Advances in phytochemical Research. Singapore: Springer; 2019: 279-298

[19] European Committee on Antimicrobial Susceptibility Testing. MIC distributions and epidemiological cut-off values (ECOFF), EUCAST SOP 10.1, 2019. Accessed October 10, 2020 at: https://www.eucast.org/ fileadmin/src/media/PDFs/EUCAST_files/EUCAST_SOPs/EUCAST_SOP_ 10.1_MIC_distributions_and_epidemiological_cut-off_value_ECOFF_ setting_20191130.pdf

[20] Beattie KD, Rouf R, Gander L, May T, Ratkowsky D, Donner C, Gill M, Grice D, Tiralongo E. Antibacterial metabolites from Australian macrofungi from the genus Cortinarius. Phytochemistry 2010; 71: 948-955

[21] Comini LR, Vieyra FEM, Mignone RA, Paez P, Mugas ML, Konigheim BS, Cabrera JL, Núñez Montoya SC, Borsarelli CD. Parietin: an efficient photo-screening pigment in vivo with good photosensitizing and photodynamic antibacterial effects in vitro. Photochem Photobiol Sci 2017; 16: $201-210$

[22] May Zin WW, Buttachon S, Dethoup T, Pereira JA, Gales L, Inácio A, Costa PM, Lee M, Sekeroglu N, Silva AMS, Pinto MMM, Kijjoa A. Antibacterial and antibiofilm activities of the metabolites isolated from the culture of the mangrove-derived endophytic fungus Eurotium chevalieri KUFA 0006. Phytochemistry 2017; 141: 86-97

[23] Malmir M, Serrano R, Silva O. Anthraquinones as potential antimicrobia Agents-a Review. In: Mendez-Vilas A, ed. Antimicrobial Research: Novel Bioknowledge and educational Programs. Badajoz: Formatex Research Center; 2017: 55-61

[24] Quang DN, Hashimoto T, Fournier J, Stadler M, Radulovic N, Asakawa Y. Sassafrins A-D, new antimicrobial azaphilones from the fungus Creosphaeria sassafras. Tetrahedron 2005; 61: 1743-1748

[25] Quang DN, Hashimoto T, Stadler M, Radulovic N, Asakawa Y. Antimicrobial azaphilones from the fungus Hypoxylon multiforme. Planta Med 2005; 71: 1058-1062

[26] Quang DN, Harinantenaina L, Nishizawa T, Hashimoto T, Kohchi C, Soma $\mathrm{Gl}$, Asakawa Y. Inhibition of nitric oxide production in RAW 264.7 cells by azaphilones from xylariaceous fungi. Biol Pharm Bull 2006; 29: 34-37

[27] Beni Z, Dekany M, Kovacs B, Csupor B, Zomborszki PZ, Kerekes EB, Szekeres A, Urbán E, Hohmann J, Ványolós A. Bioactivity-guided isola- 
tion of antimicrobial and antioxidant metabolites from the mushroom Tapinella atrotomentosa. Molecules 2018; 23: 1082

[28] Benedict RG, Brady LR. Antimicrobial activity of mushroom metabolites. J Pharm Sci 1972; 61: 1820-1822

[29] Zheng C], Sohn M], Kim WG. Atromentin and leucomelone, the first inhibitors specific to enoyl-ACP reductase (FabK) of Streptococcus pneumoniae. J Antibiot (Tokyo) 2006; 59: 808-812

[30] Łopusiewicz $Ł$. The isolation, purification and analysis of the melanin pigment extracted from Armillaria mellea rhizomorphs. World Sci News 2018; 100: 135-153

[31] Bin L, Wei L, Xiaohong C, Mei ], Dong M. In vitro antibiofilm activity of the melanin from Auricularia auricula, an edible jelly mushroom. Ann Microbiol 2012; 62: 1523-1530

[32] Chepkirui C, Cheng T, Sum WC, Matasyoh JC, Decock C, Praditya DF, Wittstein K, Steinmann E, Statdler M. Skeletocutins A-L: antibacterial agents from the Kenyan wood-inhabiting Basidiomycete, Skeletocutis sp. J Agric Food Chem 2019; 67: 8468-8475

[33] de Carvalho MP, Gulotta G, do Amaral MW, Lünsdorf H, Sasse F, Abraham WR. Coprinuslactone protects the edible mushroom Coprinus comatus against biofilm infections by blocking both quorum-sensing and MurA. Environ Microbiol 2016; 18: 4254-4264

[34] Beltran-Garcia M], Estarron-Espinosa M, Ogura T. Volatile compounds secreted by the oyster mushroom (Pleurotus ostreatus) and their antibacterial activities. J Agric Food Chem 1997; 45: 4049-4052

[35] Heleno SA, Ferreira ICFR, Esteves AP, Ćirić A, Glamočlija J, Martins A, Soković M, Queiroz MJRP. Antimicrobial and demelanizing activity of Ganoderma lucidum extract, $p$-hydroxybenzoic and cinnamic acids and their synthetic acetylated glucuronide methyl esters. Food Chem Toxicol 2013; 58: 95-100

[36] Upadhyay M, Shrivastava B, Jain A, Kidwai M, Kumar S, Gomes J, Goswami DG, Kumar Panda A, Chander Kuhad R. Production of ganoderic acid by Ganoderma lucidum RCKB-2010 and its therapeutic potential. Ann Microbiol 2014; 64: 839-846

[37] Shameem N, Kamili AN, Ahmad M, Massoodi FA, Parray JA. Antimicrobial activity of crude fractions and morel compounds from wild edible mushrooms of North western Himalaya. Microb Pathog 2017; 105: 356-360

[38] Duan YC, Feng J, Bai N, Li GH, Zhang KQ, Zhao PJ. Four novel antibacterial sesquiterpene- $\alpha$-amino acid quaternary ammonium hybrids from the mycelium of mushroom Stereum hirsutum. Fitoterapia 2018; 128: 213217

[39] Stadler M, Anke T, Dasenbrock J, Steglich W. Phellodonic acid, a new biologically active hirsutane derivative from Phellodon melaleucus (Thelephoraceae, Basidiomycetes). Z Für Naturforschung C 1993; 48: 545-549

[40] Ishikawa NK, Fukushi Y, Yamaji K, Tahara S, Takahashi K. Antimicrobial cuparene-type sesquiterpenes, enokipodins $C$ and $D$, from a mycelial culture of Flammulina velutipes. J Nat Prod 2001; 64: 932-934

[41] Birnbacher ], Schüffler A, Deininger F, Opatz T, Anke T. Isolation and biological activity of new norhirsutanes from Creolophus cirrhatus. Z Für Naturforschung C 2008; 63: 203-206

[42] Mothana RAA, Jansen R, Jülich WD, Lindequist U. Ganomycins A and B, new antimicrobial farnesyl hydroquinones from the basidiomycete Ganoderma pfeifferi. J Nat Prod 2000; 63: 416-418

[43] Nitthithanasilp S, Intaraudom C, Boonyuen N, Suvannakad R, Pittayakhajonwut P. Antimicrobial activity of cyathane derivatives from Cyathus subglobisporus BCC44381. Tetrahedron 2018; 74: 6907-6916

[44] Shiono Y, Hiramatsu F, Murayama T, Koseki T, Funakoshi T. Two cyathane-type diterpenoids from the liquid culture of Strobilurus tenacellus. Chem Biodivers 2008; 5: 1811-1816

[45] Shibata H, Irie A, Morita Y. New antibacterial diterpenoids from the Sarcodon scabrosus fungus. Biosci Biotechnol Biochem 1998; 62: 24502452
[46] Liu YP, Dai Q, Pu C], Wang M, Li ZH, Liu JK, Feng T. Psathyrellanic acid, a monocyclic diterpenoid from the Basidiomycete Psathyrella candolleana. Nat Prod Commun 2019; 14: 1-4

[47] Liu YP, Dai Q, Wang WX, He J, Li JH, Feng T, Lui JK. Psathyrins: antibacterial diterpenoids from Psathyrella candolleana. J Nat Prod 2020; 83: 1725-1729

[48] Kavanagh F, Hervey A, Robbins W]. Antibiotic substances from Basidiomycetes VIII. Pleurotus multilus (Fr.) Sacc. and Pleurotus passeckerianus Pilat. Proc Natl Acad Sci U S A 1951; 37: 570-574

[49] Hartley AJ, de Mattos-Shipley K, Collins CM, Kilaru S, Foster J, Bailey A. Investigating pleuromutilin-producing Clitopilus species and related basidiomycetes. FEMS Microbiol Lett 2009; 297: 24-30

[50] Kavanagh F, Hervey A, Robbins W]. Antibiotic substances from Basidiomycetes IX. Drosophila subatrata. (Batsch ex Fr.) Quel. Proc Natl Acad Sci U S A $1952 \cdot 38 \cdot 555-560$

[51] Paukner S, Riedl R. Pleuromutilins: potent drugs for resistant bugsmode of action and resistance. Cold Spring Harb Perspect Med 2017; 7: $\mathrm{a} 027110$

[52] Mendes RE, Paukner S, Doyle TB, Gelone SP, Flamm RK, Sader HS. Low prevalence of gram-positive isolates showing elevated lefamulin MIC results during the SENTRY surveillance program for 2015-2016 and characterization of resistance mechanisms. Antimicrob Agents Chemother 2019; 63: e02158-18

[53] File TM, Goldberg L, Das A, Sweeney C, Saviski ], Gelone SP, Seltzer E, Paukner S, Wicha WW, Talbot G, Gasink LB. Efficacy and safety of intravenous-to-oral lefamulin, a pleuromutilin antibiotic, for the treatment of community-acquired bacterial pneumonia: the phase III lefamulin evaluation against pneumonia (LEAP 1) trial. Clin Infect Dis Off Publ Infect Dis Soc Am 2019; 69: 1856-1867

[54] Al-Fatimi MA, Jülich WD, Jansen R, Lindequist U. Bioactive components of the traditionally used mushroom Podaxis pistillaris. Evid-Based Complement Altern Med ECAM 2006; 3: 87-92

[55] Herath HMTB, Jacob M, Wilson AD, Abbas HK, Dhammika Nanayakkara NP. New secondary metabolites from bioactive extracts of the fungus Armillaria tabescens. Nat Prod Res 2013; 27: 1562-1568

[56] Yuyama KT, Wendt L, Surup F, Kretz R, Chepkirui C, Wittstein K, Boonlarppradab C, Wongkanoun S, Luangsa-Ard J, Stadler M, Abraham WR. Cytochalasans act as inhibitors of biofilm formation of Staphylococcus aureus. Biomolecules 2018; 8: 129

[57] Mygind PH, Fischer RL, Schnorr KM, Hansen MT, Sönksen CP, Ludvigsen S, Raventós D, Buskov S, Christensen B, De Maria L, Taboureau O, Yaver D, Elvig-Jørgensen SG, Sørensen MV, Christensen BE, Kjaerulff S, Frimodt-Moller N, Lehrer RI, Zasloff M, Kristensen HH. Plectasin is a peptide antibiotic with therapeutic potential from a saprophytic fungus. Nature 2005; 437: 975-980

[58] Schneider T, Kruse T, Wimmer R, Wiedemann I, Sass V, Pag U, Jansen A Nielsen AK, Mygind PH, Raventós DS, Neve S, Ravn B, Bonvin AMJ], De Maria L, Andersen AS, Gammelgaard LK, Sahl HG, Kristensen HH. Plectasin, a fungal defensin, targets the bacterial cell wall precursor Lipid II. Science 2010; 328: 1168-1172

[59] Li Z, Wang X, Wang X, Teng D, Mao R, Hao Y, Wang J. Research advances on plectasin and its derivatives as new potential antimicrobial candidates. Process Biochem 2017; 56: 62-70

[60] Essig A, Hofmann D, Muench D, Gayathri S, Künzler M, Kallio PT, Sahl HG, Wider G, Schneider T, Aebi M. Copsin, a novel peptide-based fungal antibiotic interfering with the peptidoglycan synthesis. J Biol Chem 2014; 289: 34953-34964

[61] Lee SJ, Yeo WH, Yun BS, Yoo ID. Isolation and sequence analysis of new peptaibol, boletusin, from Boletus spp. J Pept Sci 1999; 5: 374-378

[62] Zheng H, Maoqing Y, Liqiu X, Wenjuan T, Liang L, Guolin Z. Purification and characterization of an antibacterial protein from the cultured mycelia of Cordyceps sinensis. Wuhan Univ J Nat Sci 2006; 11: 709-714 
[63] Houshdar Tehrani MH, Fakhrehoseini E, Kamali Nejad M, Mehregan H, Hakemi-Vala M. Search for proteins in the liquid extract of edible mushroom, Agaricus bisporus, and studying their antibacterial effects. Iran J Pharm Res 2012; 11: 145-150

[64] Ngai PHK, Ng TB. A ribonuclease with antimicrobial, antimitogenic and antiproliferative activities from the edible mushroom Pleurotus sajor-caju. Peptides 2004; 25: 11-17

[65] Adeeyo AO, Lateef A, Gueguim-Kana EB. Optimization of the production of extracellular polysaccharide from the Shiitake medicinal mushroom Lentinus edodes (Agaricomycetes) using mutation and a genetic algorithm-coupled artificial neural network (GA-ANN). Int J Med Mushrooms 2016; 18: 571-581

[66] Zhu H, Sheng K, Yan E, Quia J, Lv F. Extraction, purification and antibacterial activities of a polysaccharide from spent mushroom substrate. Int J Biol Macromol 2012; 50: 840-843

[67] Cai M, Lin Y, Luo Y, Liang HH, Sun P. Extraction, antimicrobial, and antioxidant activities of crude polysaccharides from the wood ear medicinal mushroom Auricularia auricula-judae (higher Basidiomycetes). Int J Med Mushrooms 2015; 17: 591-600

[68] Li S, Shah NP. Antioxidant and antibacterial activities of sulphated polysaccharides from Pleurotus eryngii and Streptococcus thermophilus ASCC 1275. Food Chem 2014; 165: 262-270

[69] Skalicka-Wozniak K, Szypowski ], Los R, Siwulski M, Sobieralski K, Głowniak K, Malm A. Evaluation of polysaccharides content in fruit bodies and their antimicrobial activity of four Ganoderma lucidum (W Curt.: Fr.) P. Karst. strains cultivated on different wood type substrates. Acta Soc Bot Pol 2012; 81: 17-21

[70] Sen IK, Mandal AK, Chakraborti S, Dey B, Chakraborty R, Islam SS. Green synthesis of silver nanoparticles using glucan from mushroom and study of antibacterial activity. Int J Biol Macromol 2013; 62: 439-449

[71] Thongbai B, Surup F, Mohr K, Kuhnert E, Hyde KD, Stadler M. Gymnopalynes $A$ and $B$, chloropropynyl-isocoumarin antibiotics from cultures of the Basidiomycete Gymnopus sp. J Nat Prod 2013; 76: 2141-2144
[72] Daferner M, Anke T, Hellwig V, Steglich W, Sterner O. Strobilurin M, tetrachloropyrocatechol and tetrachloropyrocatechol methyl ether: new antibiotics from a Mycena species. J Antibiot (Tokyo) 1998; 51: 816-822

[73] Takazawa H, Tajima F, Miyashita C. An antifungal compound from “Shiitake" (Lentinus edodes). Yakugaku Zasshi 1982; 102: 489-491

[74] Bailey AM, Alberti F, Kilaru S, Collins CM, de Mattos-Shipley K, Hartley A], Hayes P, Griffin A, Lazarus CM, Cox RJ, Willis CL, O’Dwyer K, Spence DW, Fostera GD. Identification and manipulation of the pleuromutilin gene cluster from Clitopilus passeckerianus for increased rapid antibiotic production. Sci Rep 2016; 6: 25202

[75] Gautam CK, Madhav M, Sinha A, Osborne W]. VIT-CM]2: Endophyte of Agaricus bisporus in production of bioactive compounds. Iran J Biotechnol 2016; 14: 19-24

[76] Quang DN, Bach DD, Hashimoto T, Asakawa Y. Chemical constituents of the Vietnamese inedible mushroom Xylaria intracolorata. Nat Prod Res 2006; 20: 317-321

[77] Feussi Tala M, Qin J, Ndongo JT, Laatsch H. New azulene-type sesquiterpenoids from the fruiting bodies of Lactarius deliciosus. Nat Prod Bioprospecting 2017; 7: 269-273

[78] Arun G, Eyini M, Gunasekaran P. Characterization and biological activities of extracellular melanin produced by Schizophyllum commune (Fries). Indian J Exp Biol 2015; 53: 380-387

[79] Schwan WR, Dunek C, Gebhardt M, Engelbrecht K, Klett T, Monte A, Toce J, Rott M, Volk TJ, LiPuma J], Liu XT, McKelvey R. Screening a mushroom extract library for activity against Acinetobacter baumannii and Burkholderia cepacia and the identification of a compound with anti-Burkholderia activity. Ann Clin Microbiol Antimicrob 2010; 9: 4

[80] Vásquez R, Rios N, Solano G, Cubilla-Rios L. Lentinoids A-D, new natura products isolated from Lentinus strigellus. Mol J Synth Chem Nat Prod Chem 2018; 23: 773-782 